\title{
Fuzzy Rough Graph Theory with Applications
}

\author{
Muhammad Akram, Maham Arshad and Shumaiza \\ Department of Mathematics, University of the Punjab, New Campus, Lahore, Pakistan* \\ E-mail:m.akram@pucit.edu.pk,mahamarshad1297@gmail.com,shumaiza00@gmail.com
}

Received 7 March 2018

Accepted 3 August 2018

\begin{abstract}
Fuzzy rough set theory is a hybrid method that deals with vagueness and uncertainty emphasized in decision-making. In this research study, we apply the concept of fuzzy rough sets to graphs. We introduce the notion of fuzzy rough digraphs and describe some of their methods of construction. In particular, we consider applications of fuzzy rough digraphs. We also present algorithms to solve decision-making problems regarding selection of a city for treatment and identification of best location in a department to set mobile phone Jammer.
\end{abstract}

Keywords: Fuzzy rough relation, Fuzzy rough digraphs, Decision- making.

\section{Introduction}

Fuzzy set theory ${ }^{23}$ introduced by Zadeh gives information about how much possibilities are there that an element belongs to the target set determined on the basis of given attribute. Fuzzy set theory is a single parameter approach. On the other hand, rough set theory ${ }^{14}$ is general mathematical approach. Rough set theory is used when we have a requirement to manipulate data on the basis of set of attributes. Rough set theory was introduced on the assumption that every object of the set is associated with a property. The objects with same property or set of properties are kept in one class. The relation generated on the basis of this similarity is a basic tool in rough set theory. A rough set consists of a pair of lower approximation and an upper approximation (of target set) determined by this relation. Lower approximation is contained in target set and upper approximation may possibly be contained in target set. In rough set theory, the objective approx- imation of each element in the target set can be interpreted as a degree that the element belongs to the target set in terms of information expressed by the given relation. The difference of upper and lower approximations is boundary region of rough set.

Dubios and Prade ${ }^{8}$ studied rough sets and fuzzy sets and they investigated that these are two different approaches to handle vagueness but are not opposite. These can be combined to obtain beneficial results. On the result of this investigation, they introduced rough fuzzy sets and fuzzy rough sets in 1990. In rough fuzzy sets, a crisp relation is used to approximate fuzzy set where as in fuzzy rough sets fuzzy relation is used. Rough relations were introduced by Pawlak ${ }^{15}$ in 1996 . Feng et al. ${ }^{9}$ introduced soft rough sets and soft rough fuzzy sets in 2010. Zhang et al. ${ }^{28}$ introduced the union and intersection operations on rough sets in 2015 . Wu et al. ${ }^{21}$ discussed approximation operators, binary relation and basis algebra in $L$-fuzzy rough sets. Zeng et al. ${ }^{26}$ considered fuzzy rough set approach for incremen-Rcieved 3

\footnotetext{
* Corresponding Author: M. Akram (makrammath@yahoo.com)
} 
tal feature selection on hybrid information systems. Dynamical updating fuzzy rough approximations for hybrid data under the variation of attribute values are studied in 25 .

Graph theory is an enormous tool in solving integrative problems in various fields including geometry, computer science, physics,optimization, operations research and social network analysis. The history of graph theory may be specifically traced to 1736 when Euler solved the correlated problem. Digraphs are more competent in these fields. A graph's relation has to be reflexive where a digraph does not has reflexive relation. If $a b$ is an edge in a graph then ba must be an edge also. In a digraph it is possible for $a b$ to be part of relation where $b a$ isn't. So digraphs are used in any situation when the flow is in one way. $\mathrm{Wu}^{20}$ introduced fuzzy digraph in 1986. Mordeson et al. ${ }^{11}$ presented operations on fuzzy graphs in 1994. Certain concepts of fuzzy graphs have been studied in ${ }^{18,22}$. Akram et al. ${ }^{3}$ presented novel applications of intuitionistic fuzzy digraphs in decision support systems. Akram et al. ${ }^{2}$ further used bipolar fuzzy digraphs in some decision support systems. Most of the set-based decision making problems have been presented using rough sets, rough fuzzy sets, generalized rough fuzzy sets, soft rough fuzzy sets and intuitionistic fuzzy soft rough sets. Akram and Zafar ${ }^{4}$ presented certain results on rough fuzzy digraphs. Zafar and Akram ${ }^{24}$ considered some applications of rough fuzzy digraphs to decision making problems. Zhan et al. ${ }^{27}$ dealt with intuitionistic fuzzy rough graphs. In this research study, we apply the concept of fuzzy rough sets to graphs. We introduce the notion of fuzzy rough digraphs and describe some of their methods of construction. In particular, we consider applications of fuzzy rough digraphs. We also present algorithms to solve decision-making problems regarding selection of a city for treatment and identification of best location in a department to set mobile phone Jammer. In the last, we present a comparative study of fuzzy rough digraphs with fuzzy digraphs.

\section{Fuzzy Rough Digraphs}

Definition 1. ${ }^{8}$ Let $U$ be a universe and $T$ a fuzzy equivalence relation on $U$. Let $A$ be fuzzy set on $U$. Then the upper and lower approximations of $A$ under $T$ denoted as $\underline{T A}$ and $\bar{T} A$ respectively, are defined as

$$
\begin{aligned}
& (\underline{T A})(x)=\bigwedge_{y \in U}[(1-T(x, y)) \vee A(y)], \\
& (\bar{T} A)(x)=\bigvee_{y \in U}[T(x, y) \wedge A(y)], \forall x \in U .
\end{aligned}
$$

The pair $(\underline{T} A, \bar{T} A)$ is called fuzzy rough set if $\bar{T} A-$ $\underline{T} A \neq \emptyset$.

Definition 2. Let $U$ be a universe and $T$ a fuzzy tolerance relation on $U$. Let $A$ be a fuzzy set on $U$ and $(\underline{T A}, \bar{T} A)$ is fuzzy rough set on $U$. Let $P^{*} \subseteq U \times U$ and $H$ fuzzy tolerance relation on $P^{*}$ such that

$H\left(x_{1} x_{2}, y_{1} y_{2}\right) \leqslant T\left(x_{1} y_{1}\right) \wedge T\left(x_{2} y_{2}\right), \forall x_{1} x_{2}, y_{1} y_{2} \in P^{*}$.

Let $P$ be fuzzy set on $P^{*}$ such that

$$
P(x y) \leqslant(\underline{T A})(x) \wedge(\underline{T A})(y), \forall x y \in P^{*} .
$$

Then the lower and upper approximations of $P$ w.r.t $H$, represented as $\underline{H} P$ and $\bar{H} P$ respectively, are defined as

$$
\begin{aligned}
& (\underline{H} P)(x y)=\bigwedge_{w z \in P^{*}}[(1-H(x y, w z)) \vee P(w z)], \\
& (\bar{H} P)(x y)=\bigvee_{w z \in P^{*}}[H(x y, w z) \wedge P(w z)], \forall x y \in P^{*} .
\end{aligned}
$$

The pair $(\underline{H} P, \bar{H} P)$ is called fuzzy rough relation.

Definition 3. A fuzzy rough digraph on a non empty set $U$ is a four ordered tuple $G=(A, T A, P, H P)$, where

(a) $T$ is a fuzzy tolerance relation on $U$,

(b) $H$ is a fuzzy tolerance relation on $P^{*} \subseteq U \times U$,

(c) $T A=(\underline{T A}, \bar{T} A)$ is a fuzzy rough set on $U$,

(d) $H P=(\underline{H} P, \bar{H} P)$ is a fuzzy rough relation on $U$,

(e) $\underline{G}=(\underline{T} A, \underline{H} P)$ and $\bar{G}=(\bar{T} A, \bar{H} P)$ are fuzzy digraphs where

$\underline{G}$ represents lower approximation of $G$ and $\bar{G}$ represents upper approximation of $G$ such that

$$
\begin{aligned}
& (\underline{H} P)(x z) \leqslant \min \{(\underline{T} A)(x),(\underline{T} A)(z)\}, \\
& (\bar{H} P)(x z) \leqslant \min \{(\bar{T} A)(x),(\bar{T} A)(z)\}, \forall x z \in P^{*} .
\end{aligned}
$$

Example 1. Let $U=\{a, b, c, d, e, f\}$ be a set and $T$ a fuzzy tolerance relation on $U$ given as in Table 1 . 
Table 1: Fuzzy tolerance relation $T$

\begin{tabular}{c|cccccc}
\hline$T$ & $a$ & $b$ & $c$ & $d$ & $e$ & $f$ \\
\hline$a$ & 1 & 0.2 & 0.3 & 0.4 & 0.5 & 0.1 \\
$b$ & 0.2 & 1 & 0.6 & 0.5 & 0.7 & 0.4 \\
$c$ & 0.3 & 0.6 & 1 & 0.8 & 0.9 & 0.3 \\
$d$ & 0.4 & 0.5 & 0.8 & 1 & 0.1 & 0.2 \\
$e$ & 0.5 & 0.7 & 0.9 & 0.1 & 1 & 0.7 \\
$f$ & 0.1 & 0.4 & 0.3 & 0.2 & 0.7 & 1 \\
\hline
\end{tabular}

Let $A$ be a fuzzy set on $U$ given by $A=$ $\{(a, 0.2),(b, 0.4),(c, 0.6),(d, 0.4),(e, 0.5),(f, 0.8)\}$. Then the lower and upper approximations of $A$ w.r.t $T$ are given by

$\underline{T} A=\{(a, 0.2),(b, 0.4),(c, 0.4),(d, 0.4),(e, 0.4),(f, 0.5)\}$, $\bar{T} A=\{(a, 0.5),(b, 0.6),(c, 0.6),(d, 0.6),(e, 0.7),(f, 0.8)\}$. It is clear that $(\underline{T A}, \bar{T} A)$ is fuzzy rough set. Let $P^{*}=$ $\{a a, a b, b c, c d, d e, e f, e b, f b\} \subseteq U \times U$. Let $P=$ $\{(a a, 0.2),(a b, 0.1),(b c, 0.3),(c d, 0.3),(d e, 0.4)$, $(e f, 0.4),(e b, 0.3),(f b, 0.2)\}$ be fuzzy set defined on $P^{*}$ and $H$ fuzzy tolerance relation on $P^{*}$ given as in Table 2.

Table 2: Fuzzy tolerance relation $H$

\begin{tabular}{c|cccccccc}
\hline$H$ & $a a$ & $a b$ & $b c$ & $c d$ & $d e$ & $e f$ & $e b$ & $f b$ \\
\hline$a a$ & 1 & 0.2 & 0.1 & 0.2 & 0.3 & 0.1 & 0.2 & 0.1 \\
$a b$ & 0.2 & 1 & 0.1 & 0.2 & 0.3 & 0.4 & 0.5 & 0.1 \\
$b c$ & 0.1 & 0.1 & 1 & 0.5 & 0.4 & 0.2 & 0.5 & 0.3 \\
$c d$ & 0.2 & 0.2 & 0.5 & 1 & 0.1 & 0.1 & 0.4 & 0.2 \\
$d e$ & 0.3 & 0.3 & 0.4 & 0.1 & 1 & 0.1 & 0.1 & 0.1 \\
$e f$ & 0.1 & 0.4 & 0.2 & 0.1 & 0.1 & 1 & 0.3 & 0.3 \\
$e b$ & 0.2 & 0.5 & 0.5 & 0.4 & 0.1 & 0.3 & 1 & 0.6 \\
$f b$ & 0.1 & 0.1 & 0.3 & 0.2 & 0.1 & 0.3 & 0.6 & 1 \\
\hline
\end{tabular}

The upper and lower approximations of $P$ are given by

$$
\begin{aligned}
\underline{H} P= & \{(a a, 0.2),(a b, 0.1),(b c, 0.3),(c d, 0.3), \\
& (d e, 0.4),(e f, 0.4),(e b, 0.3),(f b, 0.2)\}, \\
\bar{H} P= & \{(a a, 0.3),(a b, 0.4),(b c, 0.4),(c d, 0.3), \\
& (d e, 0.4),(e f, 0.4),(e b, 0.3),(f b, 0.3)\} .
\end{aligned}
$$

The fuzzy rough digraph $\mathrm{G}=(\mathrm{TA}, \mathrm{HP})$ is shown in the Fig. 1. Where $\underline{G}=(\underline{T} A, \underline{H} P)$ and $\bar{G}=(\bar{T} A, \bar{H} P)$ are lower and upper approximates of $G$.
Definition 4. Let $G=(\underline{G}, \bar{G})$ be fuzzy rough digraph on nonempty set $U$. The order of $G$, represented as $O(G)$, defined by $O(G)=O(\underline{G})+O(\bar{G})$, where

$$
\begin{aligned}
& O(\underline{G})=\sum_{x \in U}(\underline{T} A)(x), \\
& O(\bar{G})=\sum_{x \in U}(\bar{T} A)(x) .
\end{aligned}
$$

Definition 5. Let $G=(\underline{G}, \bar{G})$ be fuzzy rough digraph on nonempty set $U$. The size of $G$, represented as $S(G)$, defined by $S(G)=S(\underline{G})+S(\bar{G})$, where,

$$
\begin{aligned}
& S(\underline{G})=\sum_{w, z \in U}(\underline{H} P)(w z), \\
& S(\bar{G})=\sum_{w, z \in U}(\bar{H} P)(w z) .
\end{aligned}
$$

Example 2. Let $G$ be a fuzzy rough digraph as shown in the Fig.1. Then $O(\underline{G})=2.3, O(\bar{G})=$ 3.9 therefore, $O(G)=2.3+3.9=6.2$. Similarly, $S(\underline{G})=2.2, S(\bar{G})=2.8$ which follows that $S(G)=$ $2.2+2.8=5$.

Definition 6. Let $G_{1}=\left(\underline{G}_{1}, \bar{G}_{1}\right)$ and $G_{2}=\left(\underline{G}_{2}, \bar{G}_{2}\right)$ be two fuzzy rough digraphs on $U$. The union of $G_{1}$ and $G_{2}$ is defined as $G=G_{1} \cup G_{2}=\left(\underline{G}_{1} \cup \underline{G}_{2}, \bar{G}_{1} \cup\right.$ $\left.\bar{G}_{2}\right)$, where $\underline{G}_{1} \cup \underline{G}_{2}=\left(\underline{T} A_{1} \cup \underline{T} A_{2}, \underline{H} \underline{P}_{1} \cup \underline{H} P_{2}\right)$ and

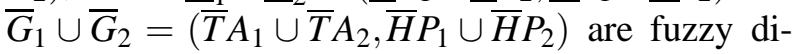
graphs, respectively, such that

(i) $\left\{\begin{array}{l}\left(\underline{T} A_{1} \cup T A_{2}\right)(w)=\max \left\{\left(T A_{1}\right)(w),\left(\underline{T} A_{2}\right)(w)\right\}, \\ \left(\bar{T} A_{1} \cup \bar{T} A_{2}\right)(w)=\max \left\{\left(\bar{T} A_{1}\right)(w),\left(\bar{T} A_{2}\right)(w)\right\}, \\ \forall w \in \operatorname{Supp}\left(A_{1} \cup A_{2}\right) .\end{array}\right.$

(ii) $\left\{\begin{aligned} & \left(\underline{H} P_{1} \cup \bar{H}_{2}\right)(w z)=\max \left\{\left(\underline{H} P_{1}\right)(w z),\left(\underline{H} P_{2}\right)(w z)\right\}, \\ & \left(\bar{H} P_{1} \cup \bar{H} P_{2}\right)(w z)=\max \left\{\left(\overline{\bar{H}} P_{1}\right)(w z),\left(\overline{\bar{H}} P_{2}\right)(w z)\right\}, \\ & \forall w z \in \operatorname{Supp}\left(P_{1} \cup P_{2}\right) .\end{aligned}\right.$

Example 3. Let $G_{1}=\left(\underline{G}_{1}, \bar{G}_{1}\right)$ and $G_{2}=\left(\underline{G}_{2}, \bar{G}_{2}\right)$ be two fuzzy rough digraphs on $U=\{a, b, c, d\}$, where $\underline{G}_{1}=\left(\underline{T} A_{1}, \underline{H} P_{1}\right)$ and $\bar{G}_{1}=\left(\bar{T} A_{1}, \bar{H} P_{1}\right)$ are fuzzy digraphs as shown in Fig. 2. 

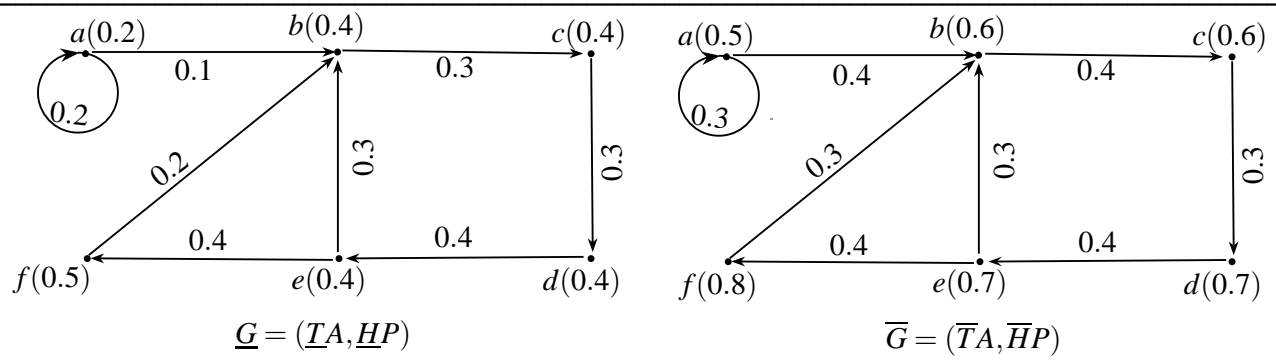

Figure 1: Lower and upper approximations of $G$
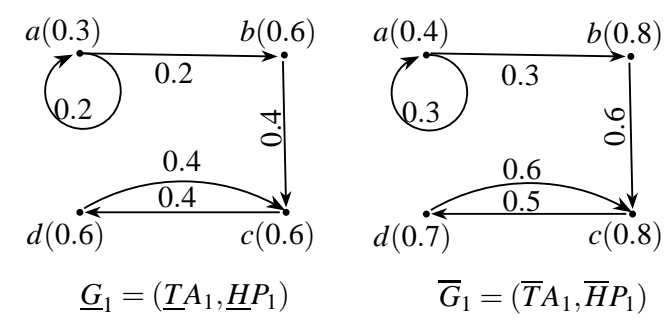

$$
\bar{G}_{1}=\left(\bar{T} A_{1}, \bar{H} P_{1}\right)
$$

Fig. 2. Lower and upper approximations of $G_{1}$

$\underline{G}_{2}=\left(\underline{T} A_{2}, \underline{H} P_{2}\right)$ and $\bar{G}_{2}=\left(\bar{T} A_{2}, \bar{H} P_{2}\right)$ are fuzzy digraphs as shown in Fig. 3.

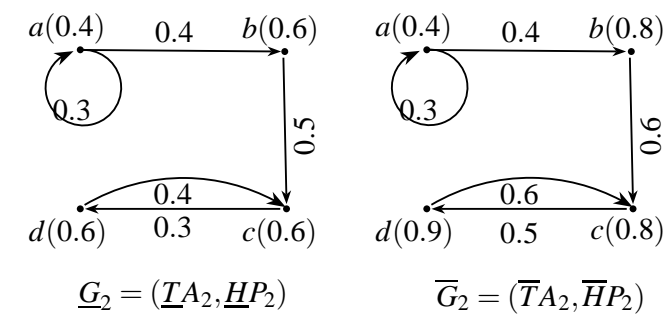

Fig. 3. Lower and upper approximations of $G_{2}$

The union of $G_{1}$ and $G_{2}$ is $G=G_{1} \cup G_{2}=\left(\underline{G}_{1} \cup\right.$ $\left.\underline{G}_{2}, \bar{G}_{1} \cup \bar{G}_{2}\right)$, where $\underline{G}_{1} \cup \underline{G}_{2}=\left(\underline{T} A_{1} \cup \underline{T} A_{2}, \underline{H} P_{1} \cup\right.$ $\left.\underline{H} P_{2}\right)$ and $\bar{G}_{1} \cup \bar{G}_{2}=\left(\bar{T} A_{1} \cup \bar{T} A_{2}, \bar{H} P_{1} \cup \bar{H} P_{2}\right)$ are fuzzy digraphs as shown in Fig. 4.

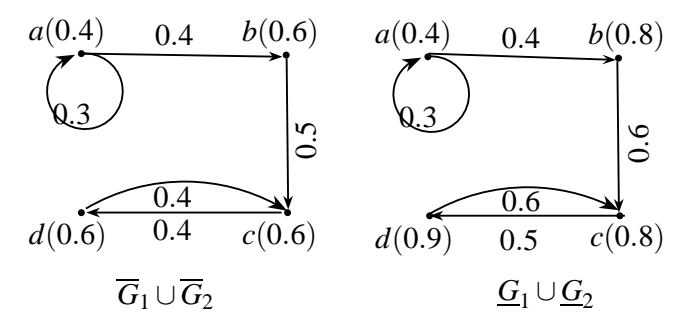

Fig. 4. Lower and upper approximations of $G_{1} \cup G_{2}$

Theorem 1. Let $G_{1}=\left(\underline{G}_{1}, \bar{G}_{1}\right)$ and $G_{2}=\left(\underline{G}_{2}, \bar{G}_{2}\right)$ be two fuzzy rough digraphs. Then $G_{1} \cup G_{2}$ is a fuzzy rough digraph.

Proof. By using similar arguments as used in the proof of Theorem 2.1 of $^{4}$, the proof is straightforward.

Definition 7. Let $G_{1}=\left(\underline{G}_{1}, \bar{G}_{1}\right)$ and $G_{2}=\left(\underline{G}_{2}, \bar{G}_{2}\right)$ be two fuzzy rough digraphs on $U$. The intersection of $G_{1}$ and $G_{2}$ is defined as $G=G_{1} \cap G_{2}=\left(\underline{G}_{1} \cap\right.$ $\left.\underline{G}_{2}, \bar{G}_{1} \cap \bar{G}_{2}\right)$, where $\underline{G}_{1} \cap \underline{G}_{2}=\left(\underline{T} A_{1} \cap \underline{T} A_{2}, \underline{H} \underline{P}_{1} \cap\right.$ $\left.\underline{H} P_{2}\right)$ and $\bar{G}_{1} \cap \bar{G}_{2}=\left(\bar{T} A_{1} \cap \bar{T} A_{2}, \bar{H} P_{1} \cap \bar{H} P_{2}\right)$ are fuzzy digraphs, respectively, such that

(i) $\left\{\begin{array}{l}\left(\underline{T} A_{1} \cap T A_{2}\right)(w)=\min \left\{\left(T A_{1}\right)(w),\left(\underline{T} A_{2}\right)(w)\right\}, \\ \left(\bar{T} A_{1} \cap \bar{T} A_{2}\right)(w)=\min \left\{\left(\bar{T} A_{1}\right)(w),\left(\bar{T} A_{2}\right)(w)\right\}, \\ \forall w \in \operatorname{Supp}\left(A_{1} \cap A_{2}\right) .\end{array}\right.$

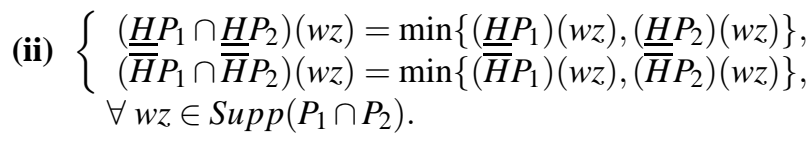

Example 4. Consider the two fuzzy rough digraphs $G_{1}$ and $G_{2}$ as shown in Fig. 2 and Fig. 3. The intersection of $G_{1}$ and $G_{2}$ is $G=G_{1} \cap G_{2}=\left(\underline{G}_{1} \cap\right.$ $\left.\underline{G}_{2}, \bar{G}_{1} \cap \bar{G}_{2}\right)$, where $\underline{G}_{1} \cap \underline{G}_{2}=\left(\underline{T} A_{1} \cap \underline{T} A_{2}, \underline{H} P_{1} \cap\right.$ 
$\left.\underline{H} P_{2}\right)$ and $\bar{G}_{1} \cap \bar{G}_{2}=\left(\bar{T} A_{1} \cap \bar{T} A_{2}, \bar{H} P_{1} \cap \bar{H} P_{2}\right)$ are fuzzy digraphs as shown in Fig. 5.
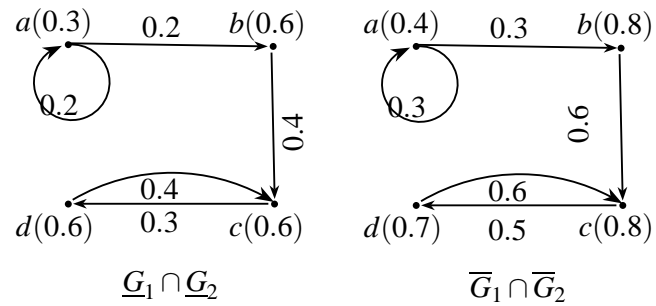

Fig. 5. Lower and upper approximations of $G_{1} \cap G_{2}$

Definition 8. Let $G_{1}=\left(\underline{G}_{1}, \bar{G}_{1}\right)$ and $G_{2}=\left(\underline{G}_{2}, \bar{G}_{2}\right)$ be two fuzzy rough digraphs on $U$. The cartesian product of $G_{1}$ and $G_{2}$ is defined as $G=G_{1} \times$ $G_{2}=\left(\underline{G}_{1} \times \underline{G}_{2}, \bar{G}_{1} \times \bar{G}_{2}\right)$, where $\underline{G}_{1} \times \underline{G}_{2}=\left(\underline{T} A_{1} \times\right.$ $\left.\underline{T} A_{2}, \underline{H} P_{1} \times \underline{H} P_{2}\right)$ and $\bar{G}_{1} \times \bar{G}_{2}=\left(\bar{T} A_{1} \times \bar{T} A_{2}, \bar{H} P_{1} \times\right.$ $\left.\overline{\bar{H}} P_{2}\right)$ are fuzzy digraphs, respectively, such that

(i) $\left\{\left(\underline{T} A_{1} \times \underline{T} A_{2}\right)(w z)=\min \left\{\left(\underline{T} A_{1}\right)(w),\left(\underline{T} A_{2}\right)(z)\right\}\right.$, $\left(\bar{T} A_{1} \times \bar{T} A_{2}\right)(w z)=\min \left\{\left(\bar{T} A_{1}\right)(w),\left(\bar{T} A_{2}\right)(z)\right\}$, $\forall w z \in \operatorname{Supp}\left(A_{1} \times A_{2}\right)$.

(ii)

$$
\left\{\begin{array}{l}
\left(\underline{H} P_{1} \times \underline{H} P_{2}\right)\left(w z_{1}, w z_{2}\right)=\min \left\{\left(\underline{\left.T A_{1}\right)(w)}\right.\right. \\
\left(\bar{H} P_{1} \times \bar{H} P_{2}\right)\left(w z_{1}, w z_{2}\right)=\min \left\{\left(\underline{\left.\underline{T} P_{2}\right)\left(z_{1}\right)(w)}\right),\right. \\
\left.\left(\bar{H} P_{2}\right)\left(z_{1} z_{2}\right)\right\} \\
\forall w \in \operatorname{Supp}\left(A_{1}\right), z_{1} z_{2} \in \operatorname{Supp}\left(P_{2}\right) .
\end{array}\right.
$$

(iii)

$$
\left\{\begin{array}{l}
\left(\underline{H} P_{1} \times \underline{H} P_{2}\right)\left(w_{1} z, w_{2} z\right)=\min \left\{\left(\underline{H} P_{1}\right)\left(w_{1} w_{2}\right),\right. \\
\left(\bar{H} P_{1} \times \bar{H} P_{2}\right)\left(w_{1} z, w_{2} z\right)=\min \left\{\left(\underline{\bar{H}} A_{2}\right)(z)\right\},\left(w_{1} w_{2}\right), \\
\left.\left(\bar{T} A_{2}\right)(z)\right\} \\
\forall w_{1} w_{2} \in \operatorname{Supp}\left(P_{1}\right), z \in \operatorname{Supp}\left(A_{2}\right) .
\end{array}\right.
$$

Example 5. Let $G_{1}=\left(\underline{G}_{1}, \bar{G}_{1}\right)$ and $G_{2}=\left(\underline{G}_{2}, \bar{G}_{2}\right)$ be two fuzzy rough digraphs on $U$ as shown in Fig. 2 and Fig. 3. The cartesian product of $G_{1}$ and $G_{2}$ is $G=G_{1} \times G_{2}=\left(\underline{G}_{1} \times \underline{G}_{2}, \bar{G}_{1} \times \bar{G}_{2}\right)$, where $G_{1} \times \underline{G}_{2}=\left(\underline{T} A_{1} \times \underline{T} A_{2}, \underline{H} \underline{P}_{1} \times \underline{H}_{2}\right)$ and $\bar{G}_{1} \times$ $\bar{G}_{2}=\left(\overline{\bar{T}} A_{1} \times \bar{T} A_{2}, \bar{H} P_{1} \times \overline{\bar{H}} P_{2}\right)$ are fuzzy digraphs as shown in Fig. 6.

Theorem 2. Let $G_{1}=\left(\underline{G}_{1}, \bar{G}_{1}\right)$ and $G_{2}=\left(\underline{G}_{2}, \bar{G}_{2}\right)$ be two fuzzy rough digraphs. Then $G_{1} \times G_{2}$ is fuzzy rough digraph.

Proof. By using similar arguments as used in the proof of Theorem 2.2 of $^{4}$, the proof is straightforward.
Definition 9. Let $G_{1}=\left(\underline{G}_{1}, \bar{G}_{1}\right)$ and $G_{2}=\left(\underline{G}_{2}, \bar{G}_{2}\right)$ be two fuzzy rough digraphs on $U$. The composition of $G_{1}$ and $G_{2}$ is defined as $G=G_{1} \circ G_{2}=\left(\underline{G}_{1} \circ\right.$ $\left.\underline{G}_{2}, \bar{G}_{1} \circ \bar{G}_{2}\right)$, where $\underline{G}_{1} \circ \underline{G}_{2}=\left(\underline{T} A_{1} \circ \underline{T} A_{2}, \underline{H} \underline{P}_{1} \circ\right.$ $\left.\underline{H}_{2}\right)$ and $\bar{G}_{1} \circ \bar{G}_{2}=\left(\bar{T} A_{1} \circ \bar{T} A_{2}, \bar{H} P_{1} \circ \bar{H} P_{2}\right)$ are fuzzy digraphs, respectively, such that

(i) $\left\{\begin{array}{l}\left(\underline{T} A_{1} \circ T A_{2}\right)(w z)=\min \left\{\left(T A_{1}\right)(w),\left(T A_{2}\right)(z)\right\} \text {, } \\ \left(\bar{T} A_{1} \circ \bar{T} A_{2}\right)(w z)=\min \left\{\left(\bar{T} A_{1}\right)(w),\left(\bar{T} A_{2}\right)(z)\right\} \text {, }\end{array}\right.$ $\forall w z \in \operatorname{Supp}\left(A_{1} \times A_{2}\right)$.

(ii) $\left\{\begin{array}{c}\left(\underline{H} P_{1} \circ \underline{H} P_{2}\right)\left(w z_{1}, w z_{2}\right)=\min \left\{\left(\underline{T} A_{1}\right)(w),\right. \\ \left(\bar{H} P_{1} \circ \bar{H} P_{2}\right)\left(w z_{1}, w z_{2}\right)=\min \left\{\left(z_{1} z_{2}\right)\right\}, \\ \left.\bar{T} A_{1}\right)(w), \\ \left.\left(\bar{H} P_{2}\right)\left(z_{1} z_{2}\right)\right\}, \\ \forall w \in \operatorname{Supp}\left(A_{1}\right), z_{1} z_{2} \in \operatorname{Supp}\left(P_{2}\right) .\end{array}\right.$

(iii) $\left\{\begin{array}{l}\left(\underline{H} P_{1} \circ \underline{H} P_{2}\right)\left(w_{1} z, w_{2} z\right)=\min \left\{\left(\underline{H} P_{1}\right)\left(w_{1} w_{2}\right),\right. \\ \left(\bar{H} P_{1} \circ \bar{H} P_{2}\right)\left(w_{1} z, w_{2} z\right)=\min \left\{\left(\bar{H} P_{1}\right)\left(w_{1} w_{2}\right),\right. \\ \left.\left(\bar{T} A_{2}\right)(z)\right\}, \\ \forall w_{1} w_{2} \in \operatorname{Supp}\left(P_{1}\right) z \in \operatorname{Supp}\left(A_{2}\right) .\end{array}\right.$

(iv) $\left\{\begin{aligned} &\left(\underline{H} P_{1} \circ \underline{H} P_{2}\right)\left(w_{1} z_{1}, w_{2} z_{2}\right)=\min \left\{\left(\underline{H} P_{1}\right)\left(w_{1} w_{2}\right),\right.\left.\left(\underline{T} A_{2}\right)\left(z_{1}\right),\left(\underline{T} A_{2}\right)\left(z_{2}\right)\right\}, \\ &\left(\bar{H} P_{1} \circ \bar{H} P_{2}\right)\left(w_{1} z_{1}, w_{2} z_{2}\right)=\min \left\{\left(\bar{H} P_{1}\right)\left(w_{1} w_{2}\right),\right. \\ &\left.\left(\bar{T} A_{2}\right)\left(z_{1}\right),\left(\bar{T} A_{2}\left(z_{2}\right)\right)\right\}, \\ & \forall w_{1} w_{2} \in \operatorname{Supp}\left(P_{1}\right) z_{1}, z_{2} \in \operatorname{Supp}\left(A_{2}\right) .\end{aligned}\right.$

Example 6. Let $G_{1}=\left(\underline{G}_{1}, \bar{G}_{1}\right)$ and $G_{2}=\left(\underline{G}_{2}, \bar{G}_{2}\right)$ be two fuzzy rough digraphs on $U$, where $\underline{G}_{1}=$ $\left(\underline{T} A_{1}, \underline{H} P_{1}\right)$ and $\bar{G}_{1}=\left(\bar{T} A_{1}, \bar{H} P_{1}\right)$ are fuzzy digraphs as shown in Fig. 7.

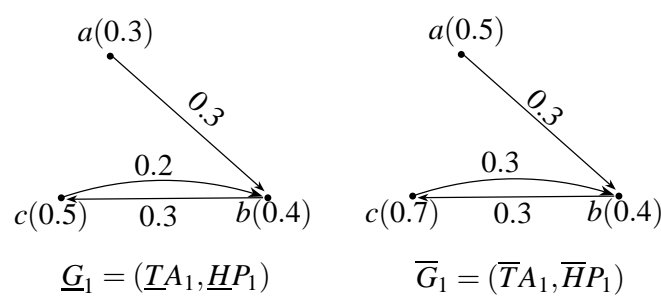

Fig. 7. Lower and upper approximations of $G_{1}$

$\underline{G}_{2}=\left(\underline{T} A_{2}, \underline{H} P_{2}\right)$ and $\bar{G}_{2}=\left(\bar{T} A_{2}, \bar{H} P_{2}\right)$ are also fuzzy graphs as shown in Fig. 8. 

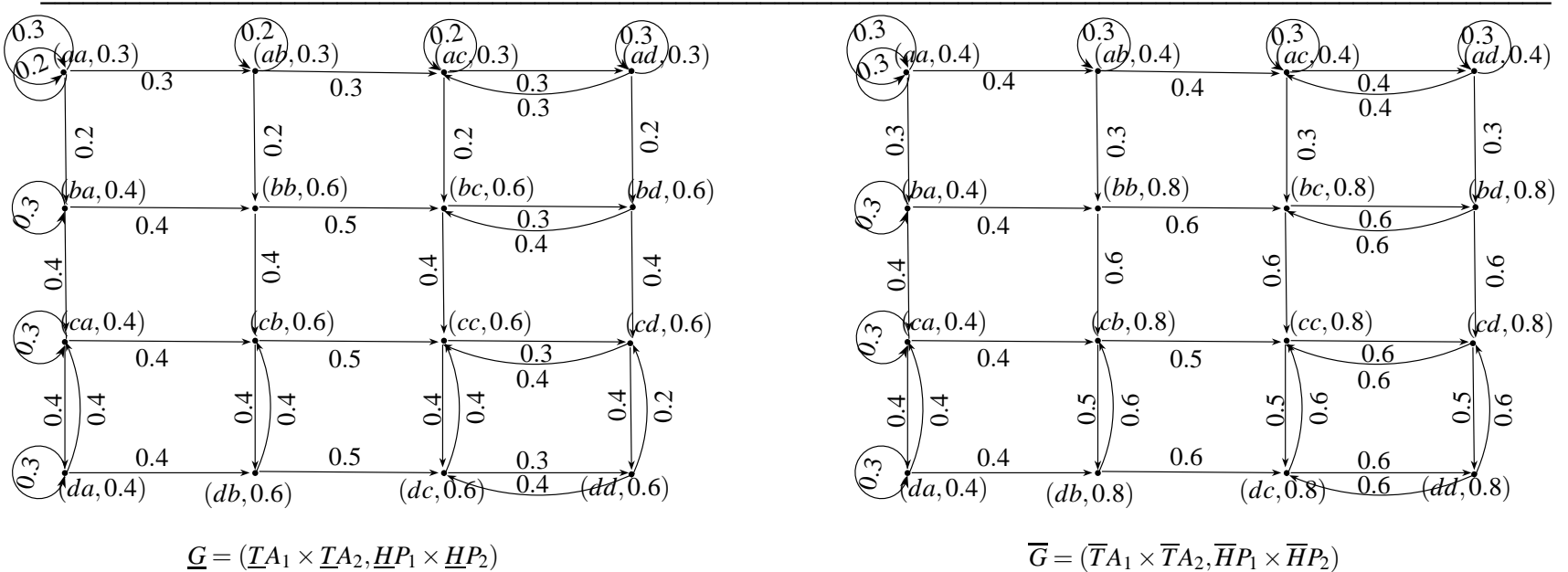

Fig. 6. Lower and upper approximations of $G_{1} \times G_{2}$
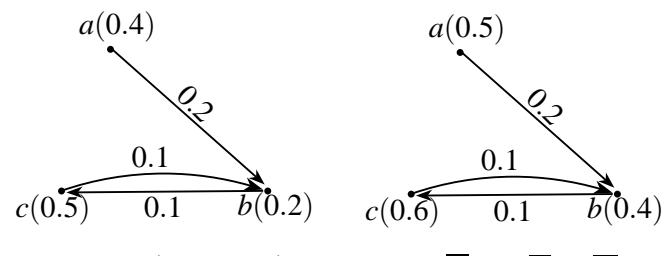

$\underline{G}_{2}=\left(\underline{T} A_{2}, \underline{H} P_{2}\right)$

$\bar{G}_{2}=\left(\bar{T} A_{2}, \bar{H} P_{2}\right)$

Fig. 8. Lower and upper approximations of $G_{2}$

The composition of $G_{1}$ and $G_{2}$ is $G=G_{1} \circ$ $G_{2}=\left(\underline{G}_{1} \circ \underline{G}_{2}, \bar{G}_{1} \circ \bar{G}_{2}\right)$, where $\underline{G}_{1} \circ \underline{G}_{2}=\left(\underline{T} A_{1} \circ\right.$ $\left.\underline{T} A_{2}, \underline{H} \bar{P}_{1} \circ \underline{H} P_{2}\right)$ and $\bar{G}_{1} \circ \bar{G}_{2}=\left(\bar{T} A_{1} \circ \bar{T} A_{2}, \bar{H} P_{1} \circ\right.$ $\left.\overline{\mathrm{H}} P_{2}\right)$ are fuzzy digraphs as shown in Fig. 9.

Theorem 3. Let $G_{1}=\left(\underline{G}_{1}, \bar{G}_{1}\right)$ and $G_{2}=\left(\underline{G}_{2}, \bar{G}_{2}\right)$ be two fuzzy rough digraphs. Then $G_{1} \circ G_{2}$ is fuzzy rough digraph.

Proof. By using similar arguments as used in the proof of Theorem 2.3 of ${ }^{4}$, the proof is straightforward.

Definition 10. Let $G=(\underline{G}, \bar{G})$ be a fuzzy rough digraph. The complement of $G$ is $G^{c}=$ $\left(\underline{G}^{c}, \bar{G}^{c}\right)$, where $\underline{G}^{c}=\left((\underline{T A})^{c},(\underline{H} P)^{c}\right)$ and $\bar{G}^{c}=$ $\left((\bar{T} A)^{c},(\bar{H} P)^{c}\right)$ are fuzzy digraphs such that

(i) $\left\{\begin{array}{l}(\underline{T} A)^{c}(w)=(\underline{T} A)(w), \\ (\bar{T} A)^{c}(w)=(\bar{T} A)(w),\end{array}\right.$ $\forall w \in U$.
Example 7. consider a fuzzy rough digraph $G$ as shown in Fig. 10.

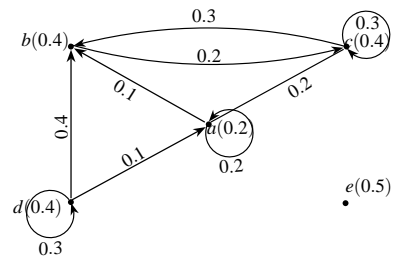

$\underline{G}=(\underline{T A}, \underline{H} P)$

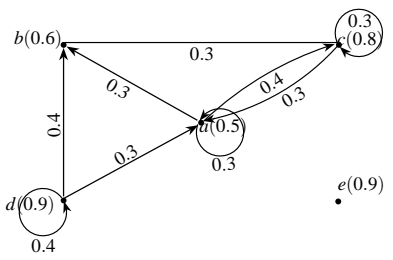

$\bar{G}=(\bar{T} A, \bar{H} P)$
Fig. 10. Lower and upper approximations of $G$

The complement of $G$ is $G^{c}=\left(\underline{G}^{c}, \bar{G}^{c}\right)$, where $\underline{G}^{c}=$ $\left((\underline{T} A)^{c},(\underline{H} P)^{c}\right)$ and $\bar{G}^{c}=\left((\bar{T} A)^{c},(\bar{H} P)^{c}\right)$ are fuzzy digraphs as shown in Fig. 11. 


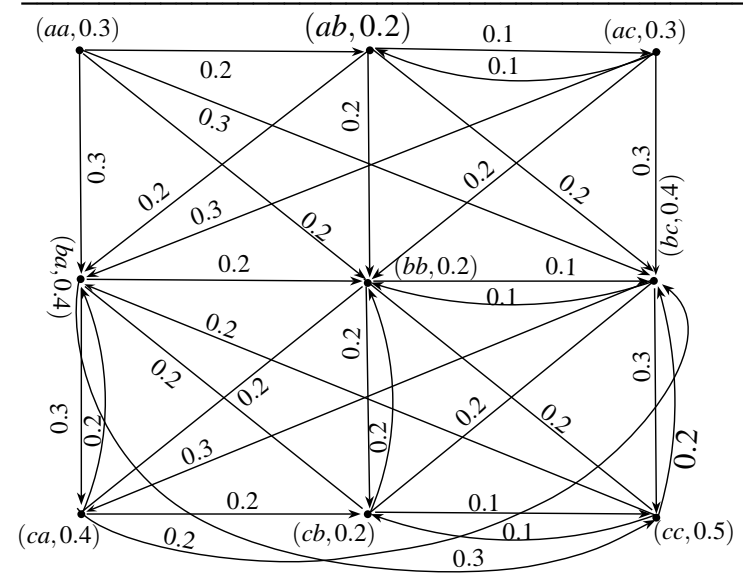

$\underline{G}_{1} \circ \underline{G}_{2}$

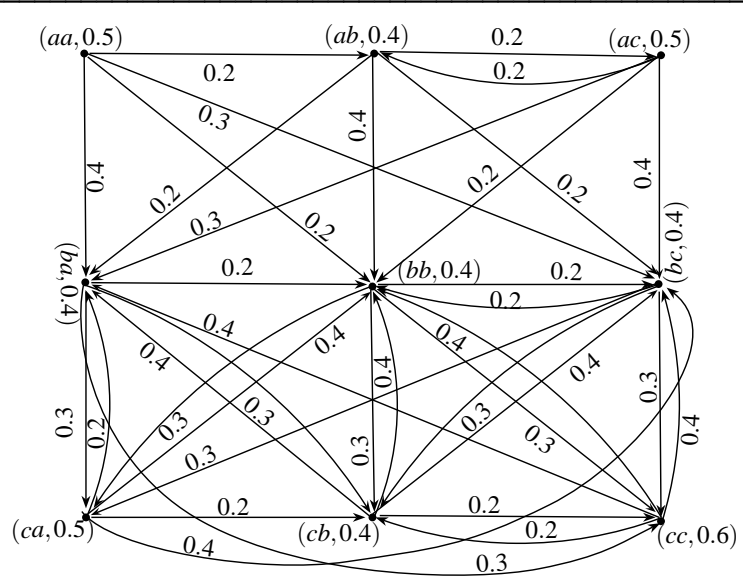

$\bar{G}_{1} \circ \bar{G}_{2}$

Fig. 9. Lower and upper approximations of $G_{1} \circ G_{2}$

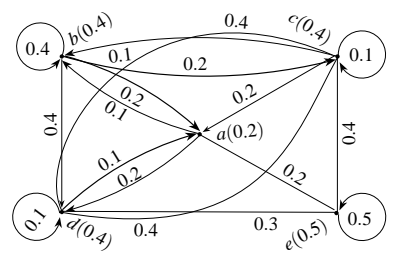

$\underline{G}^{c}=\left((\underline{T A})^{c},(\underline{H} P)^{c}\right)$

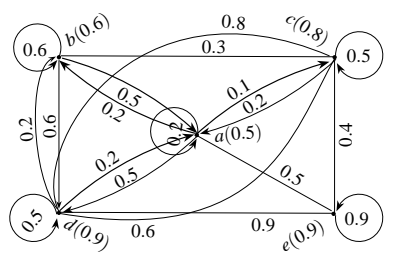

$\bar{G}^{c}=\left((\bar{T} A)^{c},(\bar{H} P)^{c}\right)$
Fig. 11. Lower and upper approximations of $G^{c}$

Definition 11. A fuzzy rough digraph is self complementary if $G$ and $G^{c}$ are isomorphic, i.e., $\underline{G} \cong \underline{G}^{c}$ and $\bar{G} \cong \bar{G}^{c}$.

Example 8. Let $U=\{a, b, c, d\}$ be a set. and $T$ a fuzzy tolerance relation on $U$ defined as in Table 3. Let $A=\{(a, 0.8),(b, 0.6),(c, 0.4),(d, 0.6)\}$ be a fuzzy set on $U$ and $T A=(\underline{T A}, \bar{T} A)$ a fuzzy rough set, where $\underline{T A}$ and $\bar{T} A$ are lower and upper approximations of $U$, respectively, as follows:

$$
\begin{aligned}
& \underline{T} A=\{(a, 0.6),(b, 0.4),(c, 0.4),(d, 0.6)\}, \\
& \bar{T} A=\{(a, 0.8),(b, 0.6),(c, 0.6),(d, 0.8)\} .
\end{aligned}
$$

Table 3. Fuzzy tolerance relation $T$

\begin{tabular}{c|cccc}
\hline$T$ & $a$ & $b$ & $c$ & $d$ \\
\hline$a$ & 1 & 0.6 & 0.4 & 0.8 \\
$b$ & 0.6 & 1 & 0.6 & 0.8 \\
$c$ & 0.4 & 0.6 & 1 & 0.4 \\
$d$ & 0.8 & 0.8 & 0.4 & 1 \\
\hline
\end{tabular}

Let $P^{*}=\{a a, a b, a c, a d, b a, b b, b c, b d, c a, c b, c c$, $c d, d a, d b, d c, d d\} \subseteq U \times U$ and $\mathrm{H}$ a fuzzy tolerance relation on $P^{*}$ defined as in Table 4. Let $P=$ $\{(a a, 0.4),(a b, 0.3),(a c, 0.3),(a d, 0.3),(b a, 0.3)$, $(b b, 0.2),(b c, 0.2),(b d, 0.2),(c a, 0.2),(c b, 0.3)$, $(c c, 0.2),(c d, 0.2),(d a, 0.3),(d b, 0.2),(d c, 0.2)$, $(d d, 0.4)\}$ be a fuzzy set on $P^{*}$ and $H P=(\underline{H} P, \bar{H} P)$ a fuzzy rough relation, where $\underline{H} P$ and $\bar{H} P$ are lower and upper approximations of $P$, respectively, as follows:

$$
\begin{aligned}
\underline{H} P= & \{(a a, 0.3),(a b, 0.2),(a c, 0.2),(a d, 0.3), \\
& (b a, 0.2),(b b, 0.2),(b c, 0.2),(b d, 0.2), \\
& (c a, 0.2),(c b, 0.2),(c c, 0.2), \\
& (c d, 0.2),(d a, 0.3),(d b, 0.2),(d c, 0.2),(d d, 0.3)\}, \\
\bar{H} P= & \{(a a, 0.4),(a b, 0.3),(a c, 0.3),(a d, 0.4), \\
& (b a, 0.3),(b b, 0.3),(b c, 0.3),(b d, 0.3), \\
& (c a, 0.3),(c b, 0.3),(c c, 0.3), \\
& (c d, 0.3),(d a, 0.4),(d b, 0.3),(d c, 0.3),(d d, 0.4)\} .
\end{aligned}
$$

Thus, $\underline{G}=(\underline{T} A, \underline{H} P)$ and $\bar{G}=(\bar{T} A, \bar{H} P)$ are fuzzy digraphs as shown in Fig. 12. 
Table 4. Fuzzy tolerance relation $H$

\begin{tabular}{c|cccccccccccccccc}
\hline$H$ & $a a$ & $a b$ & $a c$ & $a d$ & $b a$ & $b b$ & $b c$ & $b d$ & $c a$ & $c b$ & $c c$ & $c d$ & $d a$ & $d b$ & $d c$ & $d d$ \\
\hline$a a$ & 1 & 0.3 & 0.3 & 0.7 & 0.3 & 0.3 & 0.3 & 0.2 & 0.3 & 0.2 & 0.2 & 0.3 & 0.4 & 0.3 & 0.2 & 0.6 \\
$a b$ & 0.3 & 1 & 0.3 & 0.8 & 0.3 & 0.6 & 0.4 & 0.4 & 0.4 & 0.3 & 0.4 & 0.2 & 0.2 & 0.8 & 0.6 & 0.3 \\
$a c$ & 0.3 & 0.3 & 1 & 0.4 & 0.3 & 0.4 & 0.4 & 0.4 & 0.2 & 0.2 & 0.4 & 0.2 & 0.3 & 0.2 & 0.8 & 0.2 \\
$a d$ & 0.7 & 0.8 & 0.4 & 1 & 0.6 & 0.6 & 0.4 & 0.4 & 0.4 & 0.2 & 0.4 & 0.4 & 0.4 & 0.4 & 0.4 & 0.7 \\
$b a$ & 0.3 & 0.3 & 0.3 & 0.6 & 1 & 0.6 & 0.4 & 0.8 & 0.6 & 0.3 & 0.4 & 0.4 & 0.3 & 0.6 & 0.4 & 0.3 \\
$b b$ & 0.3 & 0.6 & 0.4 & 0.6 & 0.6 & 1 & 0.6 & 0.8 & 0.4 & 0.3 & 0.4 & 0.6 & 0.3 & 0.6 & 0.6 & 0.3 \\
$b c$ & 0.3 & 0.4 & 0.4 & 0.4 & 0.4 & 0.6 & 1 & 0.4 & 0.4 & 0.3 & 0.6 & 0.4 & 0.2 & 0.4 & 0.8 & 0.3 \\
$b d$ & 0.2 & 0.4 & 0.4 & 0.4 & 0.8 & 0.8 & 0.4 & 1 & 0.6 & 0.3 & 0.2 & 0.4 & 0.3 & 0.6 & 0.4 & 0.3 \\
$c a$ & 0.3 & 0.4 & 0.2 & 0.4 & 0.6 & 0.4 & 0.4 & 0.6 & 1 & 0.3 & 0.4 & 0.8 & 0.3 & 0.4 & 0.4 & 0.3 \\
$c b$ & 0.2 & 0.3 & 0.2 & 0.2 & 0.3 & 0.3 & 0.3 & 0.3 & 0.3 & 1 & 0.4 & 0.8 & 0.3 & 0.2 & 0.4 & 0.3 \\
$c c$ & 0.2 & 0.4 & 0.4 & 0.4 & 0.4 & 0.4 & 0.6 & 0.2 & 0.4 & 0.4 & 1 & 0.4 & 0.2 & 0.4 & 0.4 & 0.3 \\
$c d$ & 0.3 & 0.2 & 0.2 & 0.4 & 0.4 & 0.6 & 0.4 & 0.4 & 0.8 & 0.8 & 0.4 & 1 & 0.3 & 0.4 & 0.2 & 0.3 \\
$d a$ & 0.4 & 0.2 & 0.3 & 0.4 & 0.3 & 0.3 & 0.2 & 0.3 & 0.3 & 0.3 & 0.2 & 0.3 & 1 & 0.3 & 0.4 & 0.7 \\
$d b$ & 0.3 & 0.8 & 0.2 & 0.4 & 0.6 & 0.6 & 0.4 & 0.6 & 0.4 & 0.2 & 0.4 & 0.4 & 0.3 & 1 & 0.4 & 0.3 \\
$d c$ & 0.2 & 0.6 & 0.8 & 0.4 & 0.4 & 0.6 & 0.8 & 0.4 & 0.4 & 0.4 & 0.4 & 0.2 & 0.4 & 0.4 & 1 & 0.3 \\
$d d$ & 0.6 & 0.3 & 0.2 & 0.7 & 0.3 & 0.3 & 0.3 & 0.3 & 0.3 & 0.3 & 0.3 & 0.3 & 0.7 & 0.3 & 0.3 & 1 \\
\hline
\end{tabular}
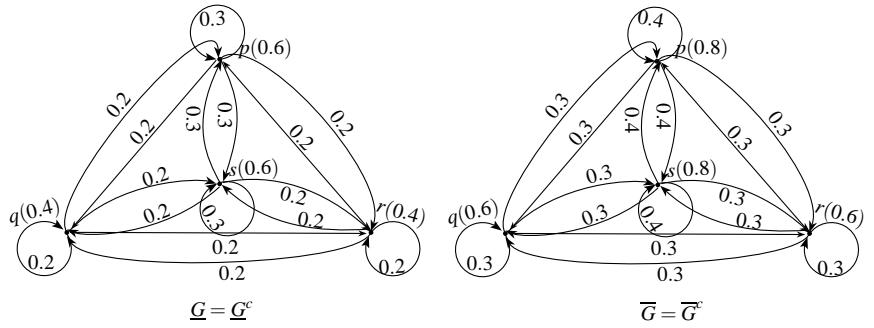

Fig. 12. Lower and upper approximations of $G^{c}$

The complement of $G$ is $G^{c}=\left(\underline{G}^{c}, \bar{G}^{c}\right)$, where $\underline{G}^{c}=$ $\underline{G}$ and $\bar{G}^{c}=\bar{G}$ are fuzzy digraphs as shown in Fig. 12 and it can be easily shown that $G$ and $G^{c}$ are isomorphic. Hence $G=(\underline{G}, \bar{G})$ is self complementary fuzzy rough digraph.

Theorem 4. Let $G=(\underline{G}, \bar{G})$ be a self complementary fuzzy rough digraph. Then

$$
\begin{aligned}
\sum_{x, z \in U}(\underline{H} P)(x z) & =\frac{1}{2} \sum_{x, z \in U}((\underline{T} A)(x) \wedge(\underline{T} A)(z)), \\
\sum_{x, z \in U}(\bar{H} P)(x z) & =\frac{1}{2} \sum_{x, z \in U}((\bar{T} A)(x) \wedge(\bar{T} A)(z)) .
\end{aligned}
$$

Proof. By using similar arguments as used in the proof of Theorem 2.4 of $^{4}$, the proof is straightforward.
Theorem 5. Let $G=(\underline{G}, \bar{G})$ be a fuzzy rough digraph. If

$$
\begin{aligned}
& (\underline{H} P)(x z)=\frac{1}{2}((\underline{T A})(x) \wedge(\underline{T A})(z)) \forall w, z \in U, \\
& (\bar{H} P)(x z)=\frac{1}{2}((\bar{T} A)(x) \wedge(\bar{T} A)(z)) \forall w, z \in U .
\end{aligned}
$$

Then $G$ is self complementary.

Proof. By using similar arguments as used in the proof of Theorem 2.5 of $^{4}$, the proof is straightforward.

Definition 12. Let $G=(\underline{G}, \bar{G})$ be a fuzzy rough digraph. The $\mu$-complement of $G$ is $G^{\mu}=$ $\left(\underline{G}^{\mu}, \bar{G}^{\mu}\right)$, where $\underline{G}^{\mu}=\left((\underline{T A})^{\mu},(\underline{H} P)^{\mu}\right)$ and $\bar{G}^{\mu}=$ $\left((\bar{T} A)^{\mu},(\bar{H} P)^{\mu}\right)$ are fuzzy digraphs such that

(i) $\left\{\begin{array}{l}(\underline{T} A)^{\mu}(w)=(\underline{T} A)(w), \\ (\bar{T} A)^{\mu}(w)=(\bar{T} A)(w),\end{array}\right.$ $\forall w \in U$.

(ii) $\quad(\underline{H} P)^{\mu}(w z)=\left\{\begin{array}{rr}\min \{(\underline{T} A)(w),(\underline{T} A)(z)\} \\ -(\underline{H} P)(w z), \\ & \text { if }(\underline{H} P)(w z)>0, \\ 0, & \text { if }(\underline{H} P)(w z)=0 .\end{array}\right.$ 


$$
(\bar{H} P)^{\mu}(w z)=\left\{\begin{aligned}
& \min \{(\bar{T} A)(w),(\bar{T} A)(z)\}-(\bar{H} P)(w z), \\
& \text { if }(\bar{H} P)(w z)>0, \\
& 0, \text { if }(\bar{H} P)(w z)=0 . \\
& \forall w, z \in U .
\end{aligned}\right.
$$

Example 9. Let $U=\{a, b, c\}$ be a set. Let $G=(\underline{G}, \bar{G})$ be a fuzzy rough digraph on $U$, where $\underline{G}=(\underline{T} A, \underline{H} P)$ and $\bar{G}=(\bar{T} A, \bar{H} P)$ are fuzzy digraphs as shown in Fig. 13.
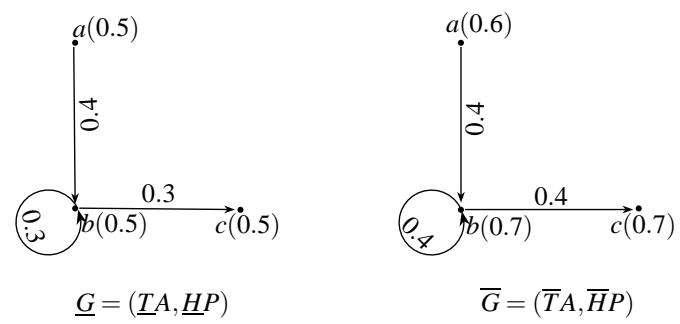

Fig. 13. Lower and upper approximations of $G$

The $\mu$-complement of $G$ is $G^{\mu}=\left(\underline{G}^{\mu}, \bar{G}^{\mu}\right)$, where $\underline{G}^{\mu}=\left((\underline{T A})^{\mu},(\underline{H} P)^{\mu}\right)$ and $\bar{G}^{\mu}=\left((\bar{T} A)^{\mu},(\bar{H} P)^{\mu}\right)$ are fuzzy digraphs as shown in Fig. 14.
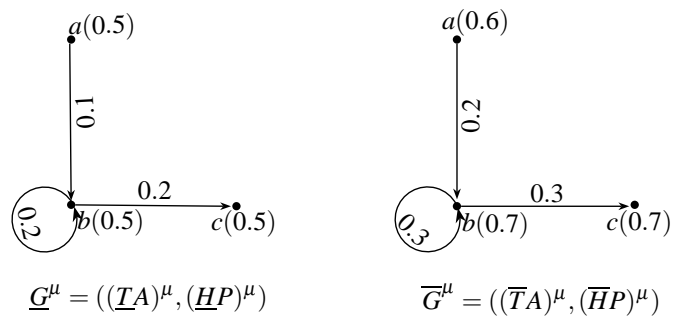

Fig. 14. Lower and upper approximations of $G^{\mu}$

Definition 13. A fuzzy rough digraph is self $\mu$-complementary if $G$ and $G^{\mu}$ are isomorphic, i.e., $\underline{G} \cong \underline{G}^{\mu}$ and $\bar{G} \cong \bar{G}^{\mu}$.

Example 10. Let $U=\{a, b, c, d\}$ be a set. and $T$ a fuzzy tolerance relation on $U$ defined as in Table 5.

\begin{tabular}{c|cccc}
\multicolumn{6}{c}{ Table 5. Fuzzy tolerance relation $T$} \\
\hline$T$ & $a$ & $b$ & $c$ & $d$ \\
\hline$a$ & 1 & 0.6 & 0.8 & 0.4 \\
$b$ & 0.6 & 1 & 0.4 & 0.6 \\
$c$ & 0.8 & 0.4 & 1 & 0.8 \\
$d$ & 0.4 & 0.6 & 0.8 & 1 \\
\hline
\end{tabular}

Let $A=\{(a, 0.7),(b, 0.6),(c, 0.8),(d, 0.4)\}$ be a fuzzy set on $U$ and $T A=(\underline{T A}, \bar{T} A)$ a fuzzy rough set, where $\underline{T A}$ and $\bar{T} A$ are lower and upper approximations of $\bar{U}$, respectively, as follows:

$$
\begin{aligned}
& \underline{T} A=\{(a, 0.6),(b, 0.4),(c, 0.4),(d, 0.4)\} \\
& \bar{T} A=\{(a, 0.8),(b, 0.6),(c, 0.8),(d, 0.8)\}
\end{aligned}
$$

Let $P^{*}=\{a a, a b, b b, a c, c a, b d, d b\} \subseteq U \times U$ and $H$ a fuzzy tolerance relation on $P^{*}$ defined as in Table 6.

Let $P=\{(a a, 0.4),(a b, 0.2),(b b, 0.2),(b c, 0.3)$, $(c c, 0.4),(c d, 0.2),(d d, 0.3),(d a, 0.2),(a c, 0.2)$, $(c a, 0.3),(b d, 0.2),(d b, 0.2)\}$ be a fuzzy set on $P^{*}$ and $H P=(\underline{H} P, \bar{H} P)$ a fuzzy rough relation, where $\underline{H} P$ and $\bar{H} P$ are lower and upper approximations of $P$, respectively, as follows:

$$
\begin{aligned}
\underline{H} P= & \{(a a, 0.3),(a b, 0.2),(b b, 0.2),(b c, 0.2),(c c, 0.2), \\
& (c d, 0.2),(d d, 0.2),(d a, 0.2),(a c, 0.2),(c a, 0.2), \\
& (b d, 0.2),(d b, 0.2)\}, \\
\bar{H} P= & \{(a a, 0.4),(a b, 0.3),(b b, 0.3),(b c, 0.3),(c c, 0.4), \\
& (c d, 0.4),(d d, 0.4),(d a, 0.4),(a c, 0.4),(c a, 0.4), \\
& (b d, 0.3),(d b, 0.3)\} .
\end{aligned}
$$

Thus, $\underline{G}=(\underline{T} A, \underline{H} P)$ and $\bar{G}=(\bar{T} A, \bar{H} P)$ are fuzzy digraphs as shown in Fig. 15.
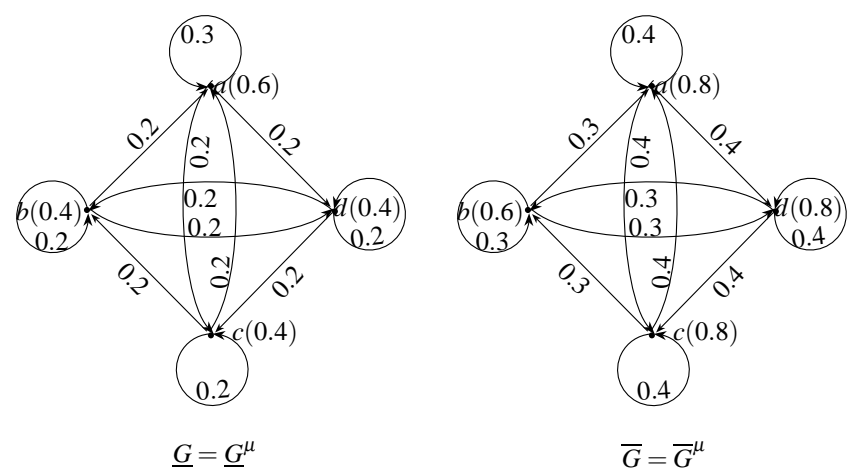

Fig. 15. Lower and upper approximations of $G^{\mu}$ 
Table 6. Fuzzy tolerance relation $H$

\begin{tabular}{c|cccccccccccc}
\hline$H$ & $a a$ & $a b$ & $b b$ & $b c$ & $c c$ & $c d$ & $d d$ & $d a$ & $a c$ & $c a$ & $b d$ & $d b$ \\
\hline$a a$ & 1 & 0.3 & 0.3 & 0.3 & 0.6 & 0.4 & 0.4 & 0.3 & 0.7 & 0.7 & 0.3 & 0.3 \\
$a b$ & 0.3 & 1 & 0.4 & 0.4 & 0.3 & 0.4 & 0.2 & 0.2 & 0.4 & 0.4 & 0.5 & 0.2 \\
$b b$ & 0.3 & 0.4 & 1 & 0.2 & 0.3 & 0.4 & 0.4 & 0.6 & 0.4 & 0.4 & 0.6 & 0.6 \\
$b c$ & 0.3 & 0.4 & 0.2 & 1 & 0.3 & 0.4 & 0.6 & 0.5 & 0.4 & 0.7 & 0.8 & 0.4 \\
$c c$ & 0.6 & 0.3 & 0.3 & 0.3 & 1 & 0.3 & 0.5 & 0.6 & 0.8 & 0.4 & 0.3 & 0.2 \\
$c d$ & 0.4 & 0.4 & 0.4 & 0.4 & 0.3 & 1 & 0.8 & 0.3 & 0.6 & 0.4 & 0.4 & 0.4 \\
$d d$ & 0.2 & 0.2 & 0.5 & 0.6 & 0.5 & 0.8 & 1 & 0.3 & 0.4 & 0.4 & 0.4 & 0.4 \\
$d a$ & 0.3 & 0.2 & 0.6 & 0.5 & 0.6 & 0.3 & 0.3 & 1 & 0.4 & 0.8 & 0.4 & 0.4 \\
$a c$ & 0.7 & 0.4 & 0.4 & 0.4 & 0.8 & 0.6 & 0.4 & 0.4 & 1 & 0.8 & 0.6 & 0.2 \\
$c a$ & 0.7 & 0.4 & 0.4 & 0.7 & 0.4 & 0.4 & 0.4 & 0.8 & 0.8 & 1 & 0.3 & 0.4 \\
$b d$ & 0.3 & 0.5 & 0.6 & 0.8 & 0.3 & 0.4 & 0.4 & 0.4 & 0.6 & 0.3 & 1 & 0.4 \\
$d b$ & 0.3 & 0.2 & 0.6 & 0.4 & 0.2 & 0.4 & 0.4 & 0.4 & 0.2 & 0.4 & 0.4 & 1 \\
\hline
\end{tabular}

The $\mu$-complement of $G$ is $G^{\mu}=\left(\underline{G}^{\mu}, \bar{G}^{\mu}\right)$, where $\underline{G}^{\mu}=\underline{G}$ and $\bar{G}^{\mu}=\bar{G}$ are fuzzy digraphs as shown in Fig. 15 and it can be easily shown that $G$ and $G^{\mu}$ are isomorphic. Hence $G=(\underline{G}, \bar{G})$ is self $\mu$-complementary fuzzy rough digraph.

We state the following results without their proofs.

Theorem 6. Let $G=(\underline{G}, \bar{G})$ be a self $\mu$-complementary fuzzy rough digraph. Then

$$
\begin{aligned}
& \sum_{(x, z) \in \underline{H} P}(\underline{H} P)(x z)=\frac{1}{2} \sum_{(x, z) \in \underline{H} P}((\underline{T} A)(x) \wedge(\underline{T} A)(z)), \\
& \sum_{(x, z) \in \bar{H} P}(\bar{H} P)(x z)=\frac{1}{2} \sum_{(x, z) \in \bar{H} P}((\bar{T} A)(x) \wedge(\bar{T} A)(z)) .
\end{aligned}
$$

Theorem 7. Let $G=(\underline{G}, \bar{G})$ be a fuzzy rough digraph. If

$$
\begin{aligned}
& (\underline{H} P)(x z)=\frac{1}{2}((\underline{T} A)(x) \wedge(\underline{T} A)(z)) \forall x, z \in U, \\
& (\bar{H} P)(x z)=\frac{1}{2}((\bar{T} A)(x) \wedge(\bar{T} A)(z)) \forall x, z \in U .
\end{aligned}
$$

Then $G$ is self $\mu$-complementary.

\section{Applications}

Decision making is very important in our daily life. There are many uncertain systems and decision making under uncertainty or the choice in uncertain environment is the central subject in many of the disciplines that are alloyed in management curriculum. Decision making is the process of identifying a problem, developing alternatives, evaluating all possible alternatives and then selecting the best one. In this section, we present an approach to decisionmaking under uncertain systems using fuzzy rough information. This method gives deep considerations of the problem as it involves lower and upper approximations of the given uncertain information.

\subsection{Selection of a city for treatment}

Emerging infectious diseases can be defined as infections that have newly appeared in a population or have existed but are rapidly increasing in incidence or geographic range. Among recent examples are Dengue fever and respiratory disease. Some infectious diseases are transmitted by bites of insects or animals and others are acquired by ingesting contaminated food. But some precautions are there that can be done to prevent from these diseases.

Consider an example of a manager of health care organization who wants to prevent the society from these infectious diseases. He has a number of cities under consideration. He collected information about emerging infectious diseases in different cities and causes of them. After investigation, he concluded that human population density is a key factor for the emergence of infectious diseases. He has a problem to choose one city that should be treated first. 
He will select that city which will have the maximum choice value among others. The problem can be represented by a fuzzy rough digraph whose vertices represent the cities and there is an edge between them if the areas joining them have increasing population density. Consider a network of eight cities $U=\left\{C_{1}, C_{2}, C_{3}, C_{4}, C_{5}, C_{6}, C_{7}, C_{8}\right\}$. Let $T$ be fuzzy tolerance relation on $U$ defined as in Table 7 .

Table 7. Fuzzy tolerance relation $T$

\begin{tabular}{c|cccccccc}
\hline$T$ & $C_{1}$ & $C_{2}$ & $C_{3}$ & $C_{4}$ & $C_{5}$ & $C_{6}$ & $C_{7}$ & $C_{8}$ \\
\hline$C_{1}$ & 1 & 0.7 & 0.8 & 0.9 & 0.6 & 0.5 & 0.7 & 0.6 \\
$C_{2}$ & 0.7 & 1 & 0.4 & 0.3 & 0.5 & 0.9 & 0.8 & 0.2 \\
$C_{3}$ & 0.8 & 0.4 & 1 & 0.5 & 0.7 & 0.6 & 0.3 & 0.4 \\
$C_{4}$ & 0.9 & 0.3 & 0.5 & 1 & 0.4 & 0.8 & 0.9 & 0.7 \\
$C_{5}$ & 0.6 & 0.5 & 0.7 & 0.4 & 1 & 0.6 & 0.5 & 0.8 \\
$C_{6}$ & 0.5 & 0.9 & 0.6 & 0.8 & 0.6 & 1 & 0.4 & 0.3 \\
$C_{7}$ & 0.7 & 0.8 & 0.3 & 0.9 & 0.5 & 0.4 & 1 & 0.9 \\
$C_{8}$ & 0.6 & 0.2 & 0.4 & 0.7 & 0.8 & 0.3 & 0.9 & 1 \\
\hline
\end{tabular}

where $T\left(C_{i}, C_{j}\right)$ represents the relationship of comparison between degree of emerging infectious diseases in $C_{i}$ and degree of emerging infectious diseases in $C_{j}$. Let $A=\left\{\left(C_{1}, 0.7\right),\left(C_{2}, 0.9\right),\left(C_{3}, 0.6\right)\right.$, $\left.\left(C_{4}, 0.5\right),\left(C_{5}, 0.6\right),\left(C_{6}, 0.7\right),\left(C_{7}, 0.8\right),\left(C_{8}, 0.9\right)\right\}$ be a fuzzy set on $U$ describing the degree of emerging infectious diseases in each city and $T A=(\underline{T A}, \bar{T} A)$ is fuzzy rough set where $T A$ and $\bar{T} A$ are lower and upper approximations of $A$ with respect to $T$ as follows:

$$
\begin{aligned}
\underline{T} A= & \left\{\left(C_{1}, 0.5\right),\left(C_{2}, 0.6\right),\left(C_{3}, 0.5\right),\left(C_{4}, 0.5\right),\left(C_{5},\right.\right. \\
& \left.0.6),\left(C_{6}, 0.5\right),\left(C_{7}, 0.5\right),\left(C_{8}, 0.5\right)\right\}, \\
\bar{T} A= & \left\{\left(C_{1}, 0.7\right),\left(C_{2}, 0.9\right),\left(C_{3}, 0.7\right),\left(C_{4}, 0.8\right),\left(C_{5},\right.\right. \\
& \left.0.8),\left(C_{6}, 0.9\right),\left(C_{7}, 0.9\right),\left(C_{8}, 0.9\right)\right\} .
\end{aligned}
$$

Let $P^{*}=\left\{C_{1} C_{2}, C_{1} C_{3}, C_{2} C_{4}, C_{3} C_{2}, C_{3} C_{5}, C_{3} C_{7}\right.$, $\left.C_{4} C_{6}, C_{4} C_{7}, C_{5} C_{7}, C_{6} C_{2}, C_{7} C_{8}, C_{8} C_{6}\right\} \subseteq U \times U$.

Let $P=\left\{\left(C_{1} C_{2}, 0.45\right),\left(C_{1} C_{3}, 0.4\right),\left(C_{2} C_{4}, 0.39\right)\right.$, $\left(C_{3} C_{2}, 0.42\right),\left(C_{3} C_{5}, 0.47\right),\left(C_{3} C_{7}, 0.35\right),\left(C_{4} C_{6}, 0.46\right)$, $\left(C_{4} C_{7}, 0.38\right),\left(C_{5} C_{7}, 0.45\right),\left(C_{6} C_{2}, 0.49\right),\left(C_{7} C_{8}, 0.43\right)$, $\left.\left(C_{8} C_{6}, 0.37\right)\right\}$ be a fuzzy set on $P^{*}$ where $P\left(C_{i}, C_{j}\right)$ $(i, j=1,2, \ldots, 8)$ represents the degree of increase in population density when we travel from $C_{i}$ towards $C_{j}$ and let $H$ be fuzzy tolerance relation on $P^{*}$ defined as in Table 8. where $H\left(C_{i} C_{j}, C_{k} C_{l}\right) C_{i}, C_{j} \in P^{*}$ represents the relationship of comparison between
$P\left(C_{i} C_{j}\right)$ and $P\left(C_{i} C_{j}\right)$. The set $H P=(\underline{H} P, \bar{H} P)$ is fuzzy rough relation where $\underline{H} P$ and $\bar{H} P$ are lower and upper approximations of $P$ with respect to $H$ as follows:

$$
\begin{aligned}
& \underline{H} P=\left\{\left(C_{1} C_{2}, 0.35\right),\left(C_{1} C_{3}, 0.4\right),\left(C_{2} C_{4}, 0.39\right),\right. \\
&\left(C_{3} C_{2}, 0.35\right),\left(C_{3} C_{5}, 0.4\right),\left(C_{3} C_{7}, 0.35\right), \\
&\left(C_{4} C_{6}, 0.4\right),\left(C_{4} C_{7}, 0.38\right),\left(C_{5} C_{7}, 0.4\right), \\
&\left.\left(C_{6} C_{2}, 0.4\right),\left(C_{7} C_{8}, 0.4\right)\left(C_{8} C_{6}, 0.37\right)\right\}, \\
& \bar{H} P=\left(C_{1} C_{2}, 0.46\right),\left(C_{1} C_{3}, 0.47\right),\left(C_{2} C_{4}, 0.45\right), \\
&\left(C_{3} C_{2}, 0.49\right),\left(C_{3} C_{5}, 0.47\right),\left(C_{3} C_{7}, 0.49\right), \\
&\left(C_{4} C_{6}, 0.49\right),\left(C_{4} C_{7}, 0.49\right),\left(C_{5} C_{7}, 0.49\right), \\
&\left.\left(C_{6} C_{2}, 0.49\right),\left(C_{7} C_{8}, 0.43\right),\left(C_{8} C_{6}, 0.46\right)\right\} .
\end{aligned}
$$

By using this fuzzy rough information, fuzzy rough digraph can be drawn as shown in Fig. 16. To identify the required city, here is required to determine a vertex which will have maximum choice value among others.

By using formula,

$\underline{(\underline{H} P} \oplus \bar{H} P)\left(C_{i} C_{j}\right)=\underline{H} P\left(C_{i}\right)+\bar{H} P\left(C_{j}\right)-\underline{H} P\left(C_{i}\right) *$ $\overline{H P}$

we have

$$
\begin{aligned}
& (\underline{H} P \oplus \bar{H} P)\left(C_{i} C_{j}\right)=\left\{\left(C_{1} C_{2}, 0.649\right),\left(C_{1} C_{3}, 0.682\right)\right. \text {, } \\
& \left(C_{2} C_{4}, 0.6645\right), \quad\left(C_{3} C_{2}, 0.6685\right), \quad\left(C_{3} C_{5}, 0.682\right), \\
& \left(C_{3} C_{7}, 0.6685\right), \quad\left(C_{4} C_{6}, 0.694\right), \quad\left(C_{4} C_{7}, 0.6838\right), \\
& \left(C_{5} C_{7}, 0.694\right), \quad\left(C_{6} C_{2}, 0.694\right), \quad\left(C_{7} C_{8}, 0.658\right), \\
& \left.\left(C_{8} C_{6}, 0.6598\right)\right\} \\
& N\left(C_{1}\right)=0.0000, \quad N\left(C_{2}\right)=2.0115, \\
& N\left(C_{3}\right)=0.6820, \quad N\left(C_{4}\right)=0.6645, \\
& N\left(C_{5}\right)=0.6820, \quad N\left(C_{6}\right)=1.3538 \text {, } \\
& N\left(C_{7}\right)=2.0463, \quad N\left(C_{8}\right)=0.6580 \text {. }
\end{aligned}
$$

$\max \{0,2.0115,0.694,0.682,0.6645,0.682$, $1.3538,2.0463,0.658\}=0.694$

Hence, $C_{7}$ is the most effected city and should be treated first.

The algorithm for determining a vertex with maximum choice value is shown in Table 9. The net time complexity of the algorithm is either $\mathrm{O}\left(n^{2} r\right)$ if $n^{2} r>r^{2}$ or $\mathrm{O}\left(r^{2}\right)$ if $n^{2} r<r^{2}$ where, $n$ is the number of vertices and $r$ is the number of edges. 


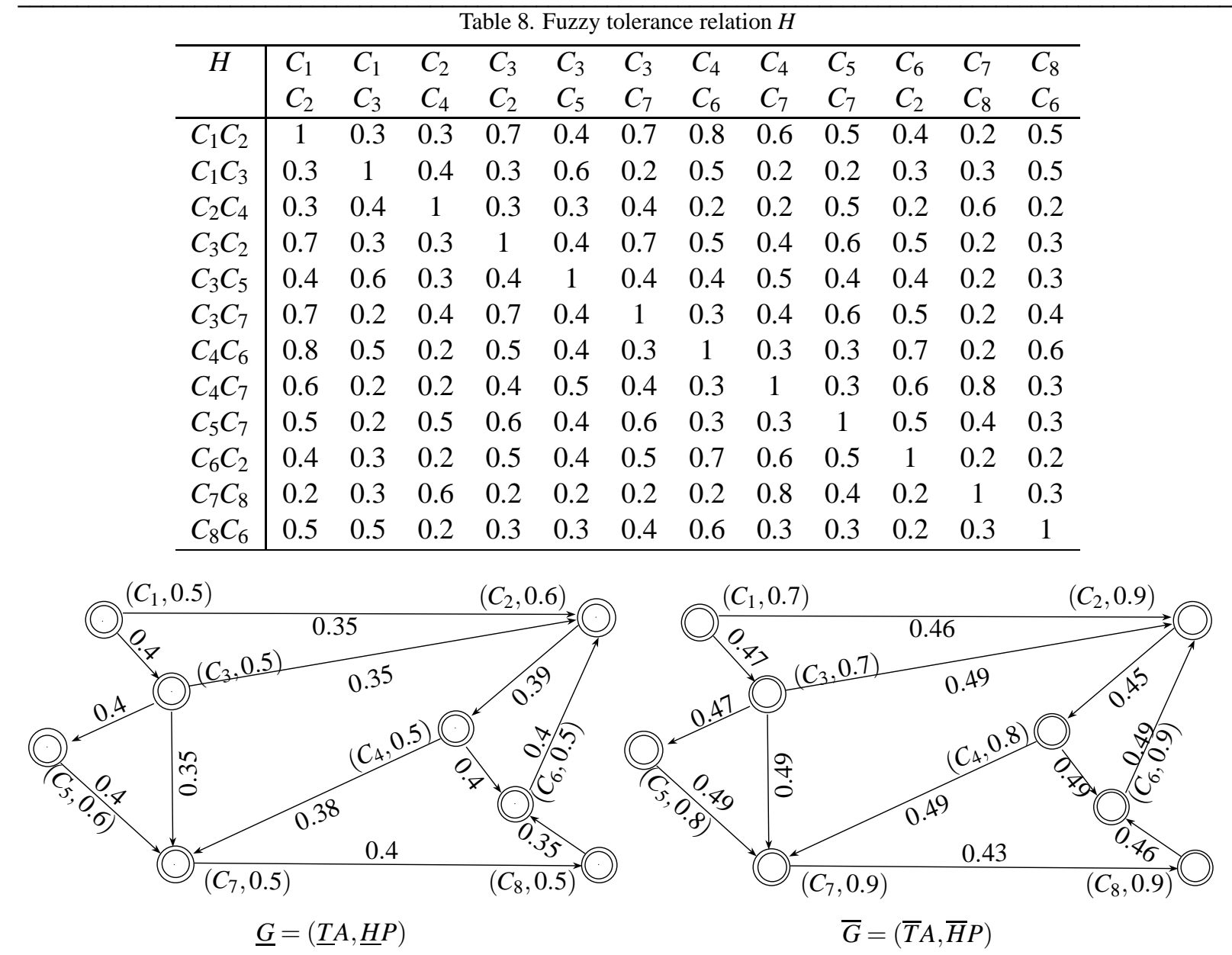

Fig. 16. Lower and upper approximations of $G$

\subsection{Identification of best location in a department to set mobile phone Jammer}

Consider an example of an institute whose director wants to set up mobile phone jammer in a number of departments in such a way that every department is in the effect of at least one of the jammer. To reduce the cost to set up strong and high quality jammer, it is required to set up minimum number of jammer. Consider a network of seven departments $U=\left\{D_{1}\right.$, $\left.D_{2}, D_{3}, D_{4}, D_{5}, D_{6}, D_{7}\right\}$.

Let $T$ be fuzzy tolerance relation on $U$ defined as in Table 10 . Where $T\left(D_{i}, D_{j}\right),(i, j=1,2, \ldots, 7)$ represents the relationship of comparison between strength of jammer in $D_{i}$ and strength of jammer in $D_{j}$.
Table 10. Fuzzy tolerance relation $T$

\begin{tabular}{c|ccccccc}
\hline$T$ & $D_{1}$ & $D_{2}$ & $D_{3}$ & $D_{4}$ & $D_{5}$ & $D_{6}$ & $D_{7}$ \\
\hline$D_{1}$ & 1 & 0.4 & 0.5 & 0.5 & 0.3 & 0.5 & 0.6 \\
$D_{2}$ & 0.4 & 1 & 0.4 & 0.5 & 0.6 & 0.6 & 0.6 \\
$D_{3}$ & 0.5 & 0.4 & 1 & 0.7 & 0.6 & 0.6 & 0.5 \\
$D_{4}$ & 0.5 & 0.5 & 0.7 & 1 & 0.4 & 0.6 & 0.6 \\
$D_{5}$ & 0.3 & 0.6 & 0.6 & 0.4 & 1 & 0.9 & 0.2 \\
$D_{6}$ & 0.5 & 0.6 & 0.6 & 0.6 & 0.9 & 1 & 0.6 \\
$D_{7}$ & 0.6 & 0.6 & 0.5 & 0.6 & 0.2 & 0.6 & 1 \\
\hline
\end{tabular}

Let $A=\left\{\left(D_{1}, 0.5\right), \quad\left(D_{2}, 0.7\right), \quad\left(D_{3}, 0.6\right)\right.$, $\left.\left(D_{4}, 0.6\right),\left(D_{5}, 0.6\right),\left(D_{6}, 0.6\right),\left(D_{7}, 0.6\right)\right\}$ be a fuzzy set on $U$ which describes the strength of jammer in each department and $T A=(\underline{T A}, \bar{T} A)$ is fuzzy rough set where $\underline{T} A$ and $\bar{T} A$ are lower and upper approxi- 
Table 9: Determining a vertex with maximum choice value

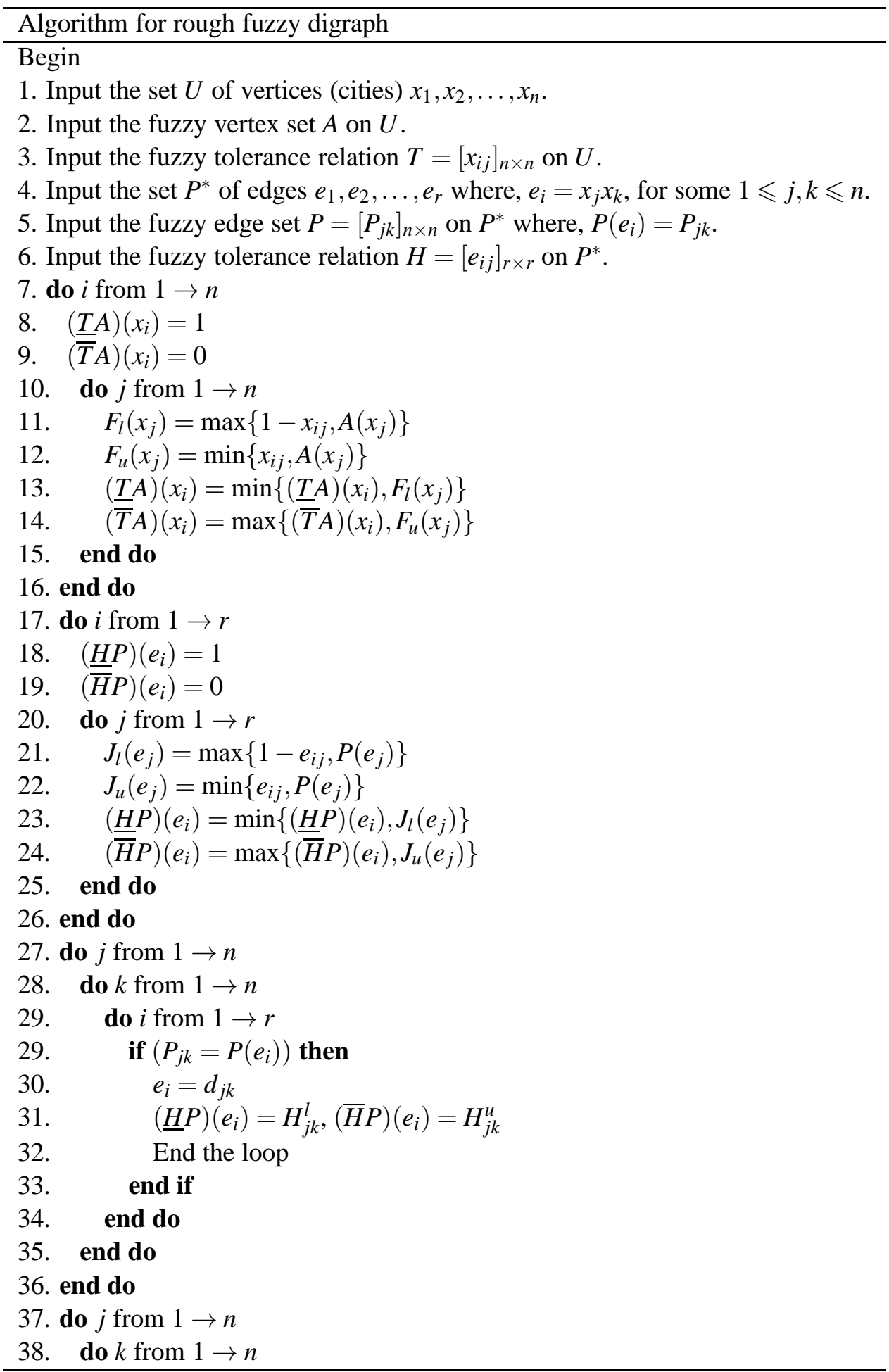




\section{Continued from previous page Table 9}

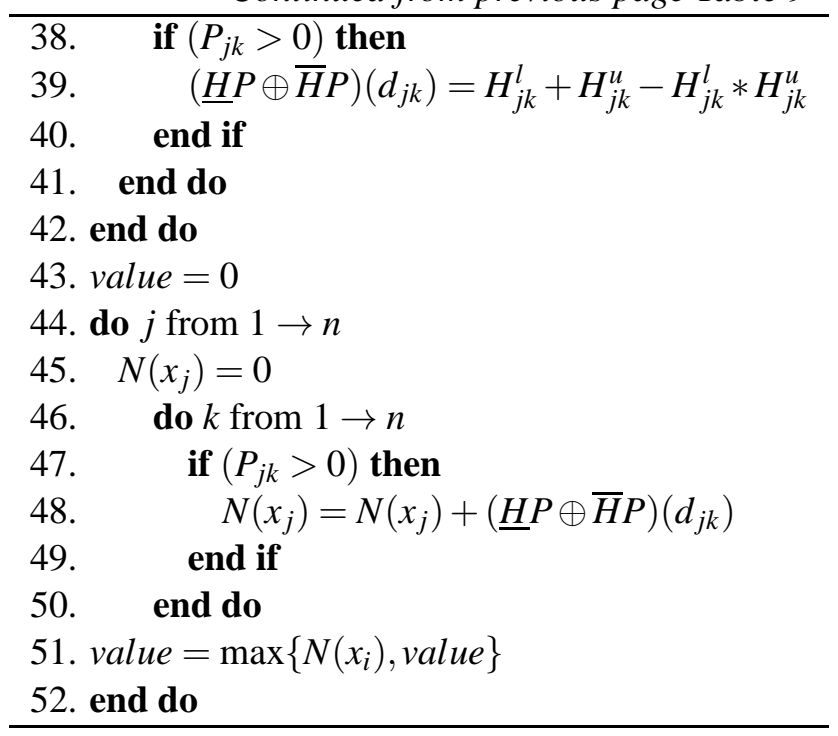

mations of $A$ with respect to $T$ as follows:

$$
\begin{aligned}
& \underline{T A}=\left\{\left(D_{1}, 0.5\right),\left(D_{2}, 0.6\right),\left(D_{3}, 0.5\right)\left(D_{4}, 0.5\right),\left(D_{5},\right.\right. \\
& \\
&\left.0.6),\left(D_{6}, 0.5\right),\left(D_{7}, 0.5\right)\right\}, \\
& \overline{T A}=\left\{\left(D_{1}, 0.6\right),\left(D_{2}, 0.7\right),\left(D_{3}, 0.6\right)\left(D_{4}, 0.6\right),\left(D_{5},\right.\right. \\
&\left.0.6),\left(D_{6}, 0.6\right),\left(D_{7}, 0.6\right)\right\} .
\end{aligned}
$$

Let $P^{*}=\left\{D_{2} D_{1}, D_{3} D_{2}, D_{3} D_{1}, D_{3} D_{4}, D_{3} D_{6}, D_{4} D_{1}\right.$, $\left.D_{5} D_{3}, D_{5} D_{6}, D_{5} D_{7}, D_{6} D_{7}\right\} \subseteq U \times U$.

Let $H$ be fuzzy tolerance relation on $P^{*}$ defined as in Table 11. $H\left(D_{i} D_{j}, D_{k} D_{l}\right) \quad D_{i} D_{j}, D_{k} D_{l} \in P^{*}$ describes the relationship of comparison between $P\left(D_{i} D_{j}\right)$ and $P\left(D_{k} D_{l}\right)$ where $P=\left\{\left(D_{2} D_{1}, 0.5\right)\right.$, $\left(D_{3} D_{2}, 0.5\right),\left(D_{3} D_{1}, 0.5\right),\left(D_{3} D_{4}, 0.5\right),\left(D_{3} D_{6}, 0.5\right)$, $\left(D_{4} D_{1}, 0.5\right),\left(D_{5} D_{3}, 0.4\right),\left(D_{5} D_{6}, 0.5\right),\left(D_{5} D_{7}, 0.6\right)$, $\left.\left(D_{6} D_{7}, 0.5\right)\right\}$ is fuzzy set on $P^{*}$ and $P\left(D_{i} D_{j}\right) \quad D_{i} D_{j}$ $\in P^{*}$ describes the degree of interference created by jammers of $D_{i}$ at the same frequency range that is used by cell phones in the surroundings of $D_{j}$. The set $H P=(\underline{H} P, \bar{H} P)$ is fuzzy rough set on $P^{*}$ where $\underline{H} P$ and $\bar{H} P$ are upper and lower approximations of $P$ with respect to $H$ as follows:

$$
\begin{aligned}
\underline{H} P=\left\{\left(D_{2} D_{1}, 0.5\right),\left(D_{3} D_{2}, 0.5\right),\left(D_{3} D_{1}, 0.5\right),\right. & \left(D_{3} D_{4}, 0.5\right),\left(D_{3} D_{6}, 0.4\right),\left(D_{4} D_{1}, 0.5\right), \\
& \left(D_{5} D_{3}, 0.4\right),\left(D_{5} D_{6}, 0.5\right),\left(D_{5} D_{7}, 0.5\right), \\
& \left.\left(D_{6} D_{7}, 0.5\right)\right\}, \\
\bar{H} P= & \left\{\left(D_{2} D_{1}, 0.5\right),\left(D_{3} D_{2}, 0.6\right),\left(D_{3} D_{1}, 0.6\right),\right.
\end{aligned}
$$

$$
\begin{aligned}
& \left(D_{3} D_{4}, 0.6\right),\left(D_{3} D_{6}, 0.5\right),\left(D_{4} D_{1}, 0.5\right), \\
& \left(D_{5} D_{3}, 0.5\right),\left(D_{5} D_{6}, 0.6\right),\left(D_{5} D_{7}, 0.6\right), \\
& \left.\left(D_{6} D_{7}, 0.5\right)\right\} .
\end{aligned}
$$

The problem can be represented by fuzzy rough digraphs as shown in Fig. 17. where vertices represent the departments and there is an edge between vertices, if one is in the effect of the gammer set up in the other. Applying the following formulae.

$$
\begin{aligned}
(\underline{T A} \oplus \bar{T} A)\left(D_{i}\right) & =\underline{T} A\left(D_{i}\right)+\bar{T} A\left(D_{i}\right) \\
& -\left(\underline{T} A\left(D_{i}\right) * \bar{T} A\left(D_{i}\right)\right) \\
(\underline{H} P \oplus \bar{H} P)\left(D_{i} D_{j}\right) & =\underline{H} P\left(D_{i} D_{j}\right)+\bar{H} P\left(D_{i} D_{j}\right) \\
& -\left(\underline{H} P\left(D_{i} D_{j}\right) * \bar{H} P\left(D_{i} D_{j}\right)\right) .
\end{aligned}
$$

It implies that,

$$
\begin{aligned}
(\underline{T} A \oplus \bar{T} A)\left(D_{i}\right)= & \left\{\left(D_{1}, 0.8\right),\left(D_{2}, 0.88\right),\right. \\
& \left(D_{3}, 0.8\right),\left(D_{4}, 0.8\right),\left(D_{5}, 0.84\right), \\
& \left.\left(D_{6}, 0.8\right),\left(D_{7}, 0.8\right)\right\}, \\
(\underline{H} P \oplus \bar{H} P)\left(D_{i} D_{j}\right)= & \left\{\left(D_{2} D_{1}, 0.75\right),\left(D_{3} D_{2}, 0.8\right),\right. \\
& \left(D_{3} D_{1}, 0.8\right),\left(D_{3} D_{4}, 0.8\right), \\
& \left(D_{3} D_{6}, 0.7\right),\left(D_{4} D_{1}, 0.75\right), \\
& \left(D_{5} D_{3}, 0.7\right),\left(D_{5} D_{6}, 0.8\right), \\
& \left.\left(D_{5} D_{7}, 0.8\right),\left(D_{6} D_{7}, 0.75\right)\right\} .
\end{aligned}
$$

Hence we have a fuzzy digraph as shown in Fig. 18. 


\begin{tabular}{cc|cccccccccc}
\hline \multicolumn{11}{c}{ Table 11. Fuzzy tolerance relation $H$} \\
\cline { 3 - 3 } & $D_{2}$ & $D_{3}$ & $D_{3}$ & $D_{3}$ & $D_{3}$ & $D_{4}$ & $D_{5}$ & $D_{5}$ & $D_{5}$ & $D_{6}$ \\
& $D_{1}$ & $D_{2}$ & $D_{1}$ & $D_{4}$ & $D_{6}$ & $D_{1}$ & $D_{3}$ & $D_{6}$ & $D_{7}$ & $D_{7}$ \\
\hline$D_{2} D_{1}$ & 1 & 0.3 & 0.3 & 0.4 & 0.4 & 0.5 & 0.5 & 0.4 & 0.2 & 0.2 \\
$D_{3} D_{2}$ & 0.3 & 1 & 0.3 & 0.4 & 0.5 & 0.3 & 0.4 & 0.5 & 0.6 & 0.3 \\
$D_{3} D_{1}$ & 0.3 & 0.3 & 1 & 0.5 & 0.5 & 0.6 & 0.5 & 0.5 & 0.6 & 0.2 \\
$D_{3} D_{4}$ & 0.4 & 0.4 & 0.5 & 1 & 0.5 & 0.5 & 0.4 & 0.5 & 0.6 & 0.3 \\
$D_{3} D_{6}$ & 0.4 & 0.5 & 0.5 & 0.5 & 1 & 0.5 & 0.6 & 0.5 & 0.2 & 0.2 \\
$D_{4} D_{1}$ & 0.5 & 0.3 & 0.6 & 0.5 & 0.5 & 1 & 0.3 & 0.3 & 0.2 & 0.2 \\
$D_{5} D_{3}$ & 0.5 & 0.4 & 0.5 & 0.4 & 0.6 & 0.3 & 1 & 0.5 & 0.4 & 0.4 \\
$D_{5} D_{6}$ & 0.4 & 0.5 & 0.5 & 0.5 & 0.5 & 0.3 & 0.5 & 1 & 0.6 & 0.2 \\
$D_{5} D_{7}$ & 0.2 & 0.6 & 0.6 & 0.6 & 0.2 & 0.2 & 0.4 & 0.6 & 1 & 0.4 \\
$D_{6} D_{7}$ & 0.2 & 0.3 & 0.2 & 0.3 & 0.2 & 0.2 & 0.4 & 0.2 & 0.4 & 1 \\
\hline
\end{tabular}

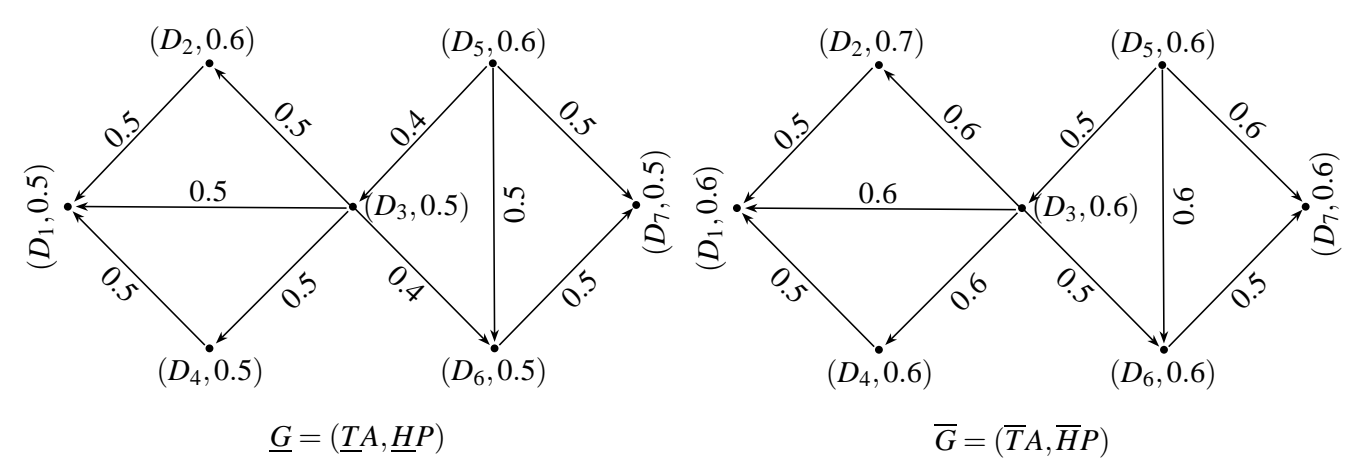

Fig. 17. Lower and upper approximations of $G$

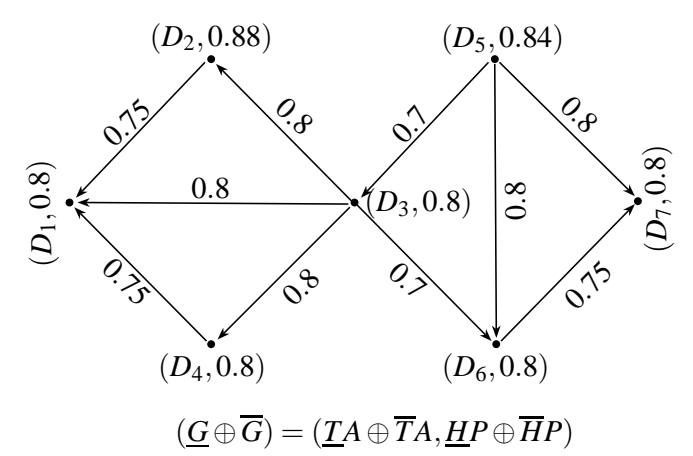

Fig. 18. Fuzzy digraph $(\underline{G} \oplus \bar{G})=(\underline{T} A \oplus \bar{T} A, \underline{H} P \oplus \bar{H} P)$

The final step is to just determine the minimal dominating set of the above digraph which will be the required solution. The dominating set is $\left\{D_{3}, D_{5}\right\}$. Hence by setting jammer only in $D_{3}$ and $D_{5}$, it can be reduced the cost.

The method of calculating a minimal dominating set is described as an algorithm in Table 12.

\section{A View of Fuzzy Rough Graphs in Comparison with Fuzzy Graphs}

The concept of fuzzy set has been utilized successfully to model uncertainty in different domains of science and technology. Due to the limitation of human knowledge to understand complex problems, it is difficult to apply single type of uncertain methods to deal with real life problems. In decisionmaking problems, it is required to consider parametric uncertainty in graphical models. For example, in the selection of a best organization for social work, we are not only interested in analyzing the working rules and characteristics of these organizations but also the evaluation of co-ordination relation between each pair of alternatives. Fuzzy rough set theory is a novel mathematical tool to overcome this difficulty. It provides lower and upper approximation of target set using fuzzy tolerance relation between any two objects. Here we present the numerical comparison 
Table 12: Algorithm for determining a minimal dominating set

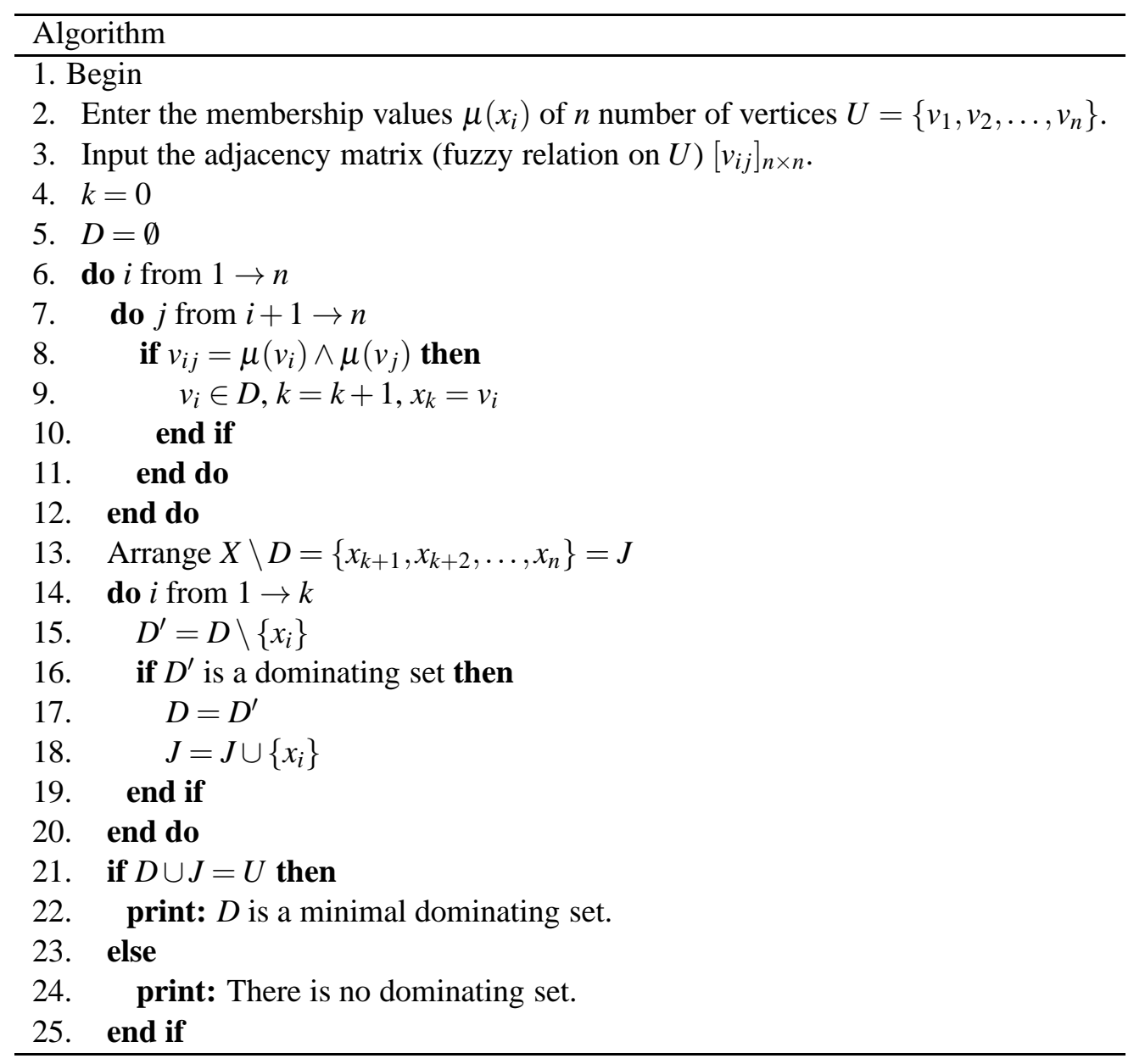

of fuzzy rough graphs with fuzzy graphs by applying fuzzy sets to above described application as follows: The problem described in subsection 3.2, can be represented using fuzzy digraphs as follows.

$$
\begin{aligned}
A= & \left\{\left(D_{1}, 0.5\right),\left(D_{2}, 0.7\right),\left(D_{3}, 0.6\right),\left(D_{4}, 0.6\right),\right. \\
& \left.\left(D_{5}, 0.6\right),\left(D_{6}, 0.6\right),\left(D_{7}, 0.6\right)\right\}
\end{aligned}
$$

can be considered as fuzzy vertex set and

$$
\begin{aligned}
P= & \left\{\left(D_{2} D_{1}, 0.5\right),\left(D_{3} D_{2}, 0.5\right),\left(D_{3} D_{1}, 0.5\right),\right. \\
& \left(D_{3} D_{4}, 0.5\right),\left(D_{3} D_{6}, 0.5\right),\left(D_{4} D_{1}, 0.5\right), \\
& \left(D_{5} D_{3}, 0.4\right),\left(D_{5} D_{6}, 0.5\right),\left(D_{5} D_{7}, 0.6\right), \\
& \left.\left(D_{6} D_{7}, 0.5\right)\right\}
\end{aligned}
$$

can be considered as fuzzy edge set. Based on fuzzy sets $A$ and $P$, the fuzzy digraph is in Fig. 19. From Fig. 19, it can be observed that to set up mobile phone jammer using given fuzzy information, we are not able to identify any location (dominating set). In this case, the fuzzy information gives no solution. To find solution of the problem, it is necessary either to change given fuzzy information or define a fuzzy tolerance relation in order to attain a suitable approximation space for finding at least one location. So, fuzzy rough set theory is more reliable in such decision-making problems. 


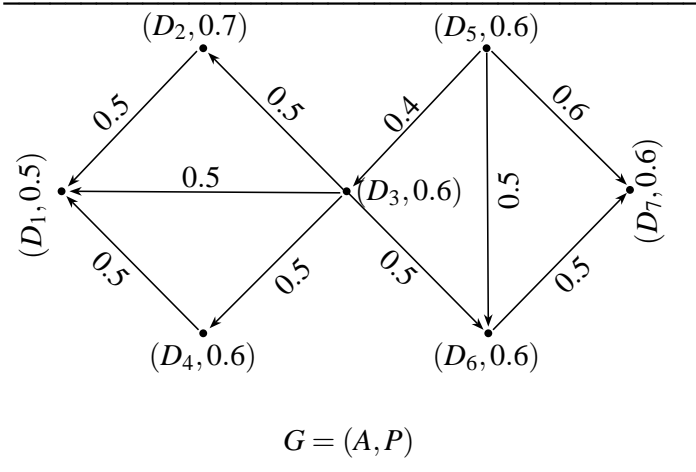

Fig. 19. Fuzzy digraph $G=(A, P)$

\section{Conclusions}

Fuzzy rough set theory gives the upper and lower approximations of a fuzzy set. In existing literature, an arbitrary and equivalence relation have been used as approximation tools in generalized rough set theory and Pawlak rough set theory, respectively. In this paper, we have developed a method using fuzzy tolerance relation as an approximation tool. We have introduced the notions of fuzzy rough relations and fuzzy rough digraphs. Fuzzy rough digraphs can be viewed as upper and lower approximation of fuzzy digraphs. We have presented fuzzy rough digraphs as an enormous tool to solve uncertain decision-making problems.

Acknowledgements: The authors are very thankful to an Associate Editor and referees for their valuable comments and suggestions for improving the paper.

\section{References}

1. M. Aggarwal, Representation of uncertainty with information and probabilistic information granules, International Journal of Fuzzy Systems (2016). doi:10.1007/s40815-016-0242-5.

2. M. Akram, N. Alshehri, B. Davvaz, A. Ashraf, Bipolar fuzzy digraphs in decision support systems, Journal of Multiple-Valued Logic and Soft Computing 27(2016) 531-551.

3. M. Akram, A. Ashraf, M. Sarwar, Novel applications of intuitionistic fuzzy digraphs in decision support systems, The Scientific World Journal 2014 Article ID 904606 (2015).

4. M. Akram, F. Zafar, Rough fuzzy digraphs with applications, Journal of Applied Mathematics and Computing, (2018)doi.org10.1007s12190-018-1171-2.

5. M. Banerjee, S.K. Pal, Roughness of a fuzzy set, Information Sciences 93(1996) 235-246.
6. P. Bhattacharya, Some remarks on fuzzy graphs, Pattern Recognition letters 6(1987) 297-302.

7. P. Corsini, V. Leoreanu-Fotea, Fuzzy sets and join spaces associated with rough sets, Rendicotni del circolo Matematico di Palermo 51(3)(2002) 527-536.

8. D. Dubois, H. Prade, Rough fuzzy sets and fuzzy rough sets, International Journal of General Systems 17(23)(1990) 191-209.

9. F. Feng, C. Li, B. Davvaz, M.I. Ali, Soft sets combined with fuzzy sets and rough sets: a tentative approach, Soft Computing 14(9)(2010) 899-911.

10. A. Kauffman, Introduction a la Theorie des Sousemsembles Flous, Masson et Cie, vol. 1, (1973).

11. J.N. Mordeson, C.S. Peng, Operations on fuzzy graphs, Information Sciences 79(1994) 159-170.

12. A. Nakamura, Fuzzy rough sets, Note Multiple-Valued Logic Japan 9(8)(1998) 1-8.

13. S. Nanda, Fuzzy rough sets, Fuzzy Sets and Systems 45(1992) 157-160.

14. Z. Pawlak, Rough sets, International Journal of Computer and Information Sciences 11(5)(1982) 341-356.

15. Z. Pawlak, Rough sets, rough relations and rough functions, Fundamenta Informaticae 27(2)(1996) 103108.

16. A. Rosenfeld, Fuzzy graphs, Fuzzy Sets and Their Applications, Academic Press New York (1975) 77-95.

17. S. Samanta and M. Pal, Fuzzy k-competition graphs and p-competition fuzzy graphs, Fuzzy Information and Engineering 5 (2013) 191-204.

18. B. Sarkar and S. Samanta, Generalized fuzzy trees, International Journal of Computational Intelligence Systems, 10 (2017) 711-720.

19. B. Z. Sun, W. Ma, Q. Liu, An approach to decision making based on intuitionistic fuzzy rough sets over two universes, Journal of Operational Research Society 64(7)(2013) 1079-1089.

20. S.Y. Wu, The compositions of fuzzy digraphs, Journal of Research in Educational Sciences 31(1986) 603628.

21. Z. Wu, T. Li, K. Qin and D. Ruan, Approximation operators, binary relation and basis algebra in L-fuzzy rough sets, Fundamenta Informaticae, 111(1)(2011)47-63.

22. Y. Yao, Y. Zhao, Attribute reduction in decisiontheoretic rough set models, Information Sciences 178(2008) 3356-3373.

23. L.A. Zadeh, Fuzzy sets, Information and control 8(3)(1965) 338-353.

24. F. Zafar, M. Akram, A novel decision-making method based on rough fuzzy information, International Journal of Fuzzy Systems, 20(3) (2018)1000-1014.

25. A. Zeng, T. Li, J. Hu, H. Chen and C. Luo, Dynamical updating fuzzy rough approximations for hybrid data under the variation of attribute values, Information Sciences, 378(2017) 363-388. 
26. A. Zeng, T. Li, D. Liu, J. Zhang and H. Chen, A fuzzy rough set approach for incremental feature selection on hybrid information systems, Fuzzy Sets and Systems, 258(2015) 39-60.

27. J. Zhan, H. M. Malik and M. Akram, Novel decisionmaking algorithms based on intuitionistic fuzzy rough environment, International Journal of Machine Learn- ing and Cybernetics, DOI: 10.1007/s13042-018-08274, 2018.

28. X. Zhang, J. Dai, Y. Yu, On the union and intersection operations of rough sets based on various approximation spaces, Information Sciences 292(2015) 214-229. 